\title{
Development of the Entorhinal Cortex Occurs via Parallel Lamination During Neurogenesis
}

Yong Liu't, Tobias Bergmann ${ }^{1 \dagger}$, Yuki Mori'2, Juan Miguel Peralvo Vidal' ${ }^{1}$, Maria Pihl' ${ }^{3}$, Navneet A. Vasistha ${ }^{4}$, Preben Dybdahl Thomsen ${ }^{3}$, Stefan E. Seemann ${ }^{5}$, Jan Gorodkin ${ }^{5}$, Poul Hyttel ${ }^{3}$, Konstantin Khodosevich ${ }^{4}$, Menno P. Witter ${ }^{6}$ and Vanessa Jane Hall ${ }^{1 *}$

${ }^{1}$ Group of Brain Development and Disease, Section Pathobiological Sciences, Department of Veterinary and Animal Sciences, Faculty of Health and Medical Sciences, University of Copenhagen, Copenhagen, Denmark, ${ }^{2}$ Center for Translational Neuromedicine, Faculty of Health and Medical Sciences, University of Copenhagen, Copenhagen, Denmark, ${ }^{3}$ Disease Stem Cell Models and Embryology, Section Pathobiological Sciences, Department of Veterinary and Animal Sciences, Faculty of Health and Medical Sciences, University of Copenhagen, Copenhagen, Denmark, ${ }^{4}$ Biotech Research and Innovation Centre, Faculty of Health and Medical Sciences, University of Copenhagen, Copenhagen, Denmark, ${ }^{5}$ Center for non-coding RNA in Technology and Health, Department of Veterinary and Animal Sciences, Faculty of Health and Medical Sciences, University of Copenhagen, Copenhagen, Denmark, ${ }^{6}$ Kavli Institute for Systems Neuroscience, Faculty of Medicine and Health Sciences, Norwegian University of Science and Technology, Trondheim, Norway

\section{OPEN ACCESS}

Edited by: Ricardo Insausti, University of Castilla-La Mancha,

Spain

Reviewed by:

Robert Francis Hevner, University of California, San Diego, United States

Sandra Cebada-Sánchez, University of Castilla-La Mancha, Spain

*Correspondence:

Vanessa Jane Hall vh@sund.ku.dk

${ }^{\dagger}$ These authors have contributed equally to this work

Received: 03 February 2021 Accepted: 08 April 2021 Published: 05 May 2021

Citation:

Liu Y, Bergmann T, Mori Y, Peralvo Vidal JM, Pihl M, Vasistha NA,

Thomsen PD, Seemann SE, Gorodkin J, Hyttel P, Khodosevich K,

Witter MP and Hall VJ (2021) Development of the Entorhinal Cortex Occurs via Parallel Lamination During Neurogenesis.

Front. Neuroanat. 15:663667. doi: 10.3389/fnana.2021.663667
The entorhinal cortex $(E C)$ is the spatial processing center of the brain and structurally is an interface between the three layered paleocortex and six layered neocortex, known as the periarchicortex. Limited studies indicate peculiarities in the formation of the EC such as early emergence of cells in layers (L) II and late deposition of LIII, as well as divergence in the timing of maturation of cell types in the superficial layers. In this study, we examine developmental events in the entorhinal cortex using an understudied model in neuroanatomy and development, the pig and supplement the research with BrdU labeling in the developing mouse EC. We determine the pig serves as an excellent anatomical model for studying human neurogenesis, given its long gestational length, presence of a moderate sized outer subventricular zone and early cessation of neurogenesis during gestation. Immunohistochemistry identified prominent clusters of OLIG2+ oligoprogenitor-like cells in the superficial layers of the lateral EC $(\mathrm{LEC})$ that are sparser in the medial EC (MEC). These are first detected in the subplate during the early second trimester. MRI analyses reveal an acceleration of EC growth at the end of the second trimester. BrdU labeling of the developing MEC, shows the deeper layers form first and prior to the superficial layers, but the LV/VI emerges in parallel and the LII/III emerges later, but also in parallel. We coin this lamination pattern parallel lamination. The early born Reln ${ }^{+}$stellate cells in the superficial layers express the classic LV marker, Bcl11b (Ctip2) and arise from a common progenitor that forms the late deep layer LV neurons. In summary, we characterize the developing EC in a novel animal model and outline in detail the formation of the EC. We further provide insight into how the periarchicortex forms in the brain, which differs remarkably to the inside-out lamination of the neocortex.

Keywords: entorhinal cortex, development, pig, diffusion tensor imaging, cortical lamination, periarchicortex, stellate cell, Reelin 


\section{INTRODUCTION}

The pig is an untraditional model in developmental neuroscience, but there are good reasons that warrant the use of the pig as a model, given its remarkable similarities to human neurodevelopment. Porcine fetal brain tissue can be easily obtained at specific time points of gestation when using controlled insemination procedures, which can be facilitated at pig production farms or in other controlled settings. Anatomically, the pig develops a gyrencephalic brain similar to humans, midway through gestation. Atlases (both annotated, unannotated and MRI) of the developing and adult pig brain are also readily available and accessible from open sources (Saikali et al., 2010; Winter et al., 2011; Conrad et al., 2012; Conrad et al., 2014). The pig brain increases significantly in size following birth, which is also reported in humans (Conrad et al., 2012). Furthermore, there is limited proliferation of neurons in the postnatal large domestic pig brain, which is also observed in the postnatal human brain (Jelsing et al., 2006). Pig gestational development is relatively long, at 114 days, which is more comparable to human gestational length (268 days) (Jukic et al., 2013) than mouse gestational length (20 days). Given the reported divergence in cortex functions between rodents and humans (Farr et al., 1988) and variations in the connectivity of the sensory cortical networks in the hippocampal region (Bergmann et al., 2016), it is necessary to consider other mammalian models which are more similar to human. Interestingly, the pig diverges from humans at an evolutionarily more distant common ancestor compared to mouse (Kumar and Hedges, 1998), but retains higher genomic similarity to humans, than mice (Wernersson et al., 2005). To sum, the developing pig brain, albeit understudied in neurodevelopment, constitutes anatomically, a particularly useful model for modeling the developing human brain.

The structure of the entorhinal cortex (EC) in the fully formed brain presents an interesting interface between the three layered paleocortex and six layered neocortex, called the periarchicortex. The EC is anatomically divided into six layers in all studied mammalian species (Dolorfo and Amaral, 1998; Insausti et al., 2017; Witter et al., 2017; Garcia and Buffalo, 2020). However, the EC layer (L)IV in the EC is cell sparse and is better known as the lamina dissecans (Insausti et al., 2017). The cell sparse LIV is a feature seen in all the allocortex subregions, including the paleocortex, archicortex and periallocortex (Stephan and Andy, 1970; Stephan, 1975, 1983; Insausti et al., 2017). Whilst the paleocortex is comprised of three layers, the archicortex and the periallocortex (the latter is further subdivided into the periarchicortex and peripaleocortex) contains four layers (Stephan and Andy, 1970; Stephan, 1975, 1983; Insausti et al., 2017). The EC has been subdivided into several areas but is now commonly divided into two, the lateral entorhinal cortex (LEC) and the medial entorhinal cortex (MEC) which deviates in both connectivity and function (Witter et al., 2017). The MEC is predominantly involved in spatial processing, whereas the LEC is predominantly involved in object recognition, odor discrimination and episodic memory (Staubli et al., 1984; Schultz et al., 2015; Vandrey et al., 2020). In the human, the EC is located in the anterior portion of the medial temporal lobe (Insausti et al., 2017), whereas, in the rat, it forms the ventrocaudal part of the cerebral hemisphere (Insausti et al., 1997). Its location in annotated pig atlases indicates it lies in the piriform lobe, although the borders and boundaries have not been well annotated. The cell types within both the MEC and LEC are well characterized anatomically in other species and many cells have been profiled by electrophysiology (Canto and Witter, 2012a,b). The principal neurons that reside in LII have received most attention. The LII comprises clustered pyramidal neurons expressing Calbindin (Calb) and scattered stellate cells in the MEC and fan cells in the LEC, both expressing Reelin (Reln) with subpopulations of pyramidal neurons, stellate cells, fan cells and multipolar neurons expressing both Calb and Reln (Witter et al., 2017). The stellate cells and pyramidal neurons in MEC LII contribute to the spatially modulated group of grid cells (Domnisoru et al., 2013; Schmidt-Hieber and Hausser, 2013; Rowland et al., 2018), whereas the fan cells in the LEC are important for object-place-context associations (Vandrey et al., 2020).

The developing entorhinal cortex has only been studied to a limited extent. The medial EC is structurally organized already by embryonic day (E)18 in the rat (Bayer, 1980a; Bayer et al., 1993) and has formed, at least rostromedially, by gestational week (GW) 13-13.5 in the human (Kostovic et al., 1993). The maturation of the MEC has been studied during gestation and postnatal development via assessment in changes of molecular markers such as Calb, Doublecortin and Wolframin across the developing MEC (Ray and Brecht, 2016; Donato et al., 2017). The findings from these studies suggest the MEC circuitry matures along the dorsal to ventral axis, at least in the superficial layers. The stellate cells are one of the first cell types to be born and their maturation drives the maturation of the hippocampal and entorhinal circuitry ending in maturation of LII in the LEC (Ray and Brecht, 2016; Donato et al., 2017). Another study has characterized the expression of a few neuronal markers across the layers of the human EC at GW 20-26 (Zykin et al., 2018), identifying Reln ${ }^{+}$Cajal Retzius cells in LI but no Reln expression in the LII neurons. Differences were also observed in MAP2 expression. MAP2 ${ }^{+}$neurons could be clearly identified in only LIII of the LEC, but not in the MEC and LV of both the MEC and LEC. A more thorough investigation of neuronal markers is required during EC development as it is not clear, for example, if Reln is switched on in stellate cells at a later time point and what other markers can be used to delineate the different layers in the developing EC.

Evidence suggests that the EC may not form in the traditional inside-out lamination pattern as the rest of the neocortex. For example, an early study revealed through thymidine autoradiography in the developing rat brain, that LII formed earlier than LIII (Bayer et al., 1993). This has to some extent been confirmed by a more recent study by Donato et al. (2017) showing the birth of stellate cells early on during EC neurogenesis in mice. However, a detailed analysis of the lamination events during neurogenesis in the EC is lacking. Reln is thought to instigate a "detach and go" mechanism which instructs newborn neurons to detach from radial glia and signals them to translocate 
to the top of the cortical plate (Cooper, 2008). Given Reln is found in both subsets of the LII and LIII neurons in the EC, it is unclear how this might affect cortical lamination of Reln ${ }^{-}$neurons in the same layers.

In this study, we sought to investigate the anatomical boundaries of the EC in the postnatal and developing EC in the pig to improve anatomical knowledge in a species that is understudied in neuroscience. We characterize the development of the EC using a large number of neural markers and investigate the pattern of cortical lamination in the EC, which forms part of the periallocortex, that differs in layer structure to the neocortex. Finally, we study the connectivity of the postnatal pig EC to elucidate its similarity to the more well studied rodents. We determine that the MEC develops by parallel lamination of the deep layers LV/VI followed by parallel lamination of the superficial layers LII/III.

\section{MATERIALS AND METHODS}

\section{Animal Welfare and Collection of Brains}

The experiments conform to the relevant regulatory standards for use of fetal material. Crossbred male and female pig fetuses at E26, E33, E39, E50, E60, E70, E80, and E100 of development were obtained from inseminated Danish Landrace/Yorkshire sows with Duroc boar semen from a local pig production farm with D0 counted as day of insemination. Deceased postnatal pigs were obtained at P75 as a gift from Per Torp Sangild at the University of Copenhagen. Adult brains were obtained from sows killed for another study using an overdose of sodium phenobarbital by a professional issued with a license from the Danish Animal Experiment Inspectorate. Numbers of porcine brains used are shown in Supplementary Table 1. Mouse experiments were carried out in accordance with the guidelines of the National Animal Ethics Committee of Denmark. In the present study, C57BL/6 wild-type mice (Janvier Labs) were used. The mice were housed in individually ventilated cages in a reversed light cycle and with free access to food and water. The number of mouse brains used are shown in Supplementary Table 2.

\section{BrdU Labeling of Mouse Brains}

Males and females were housed together until a vaginal plug (VP) was detected, after which the males were separated. Detection of the VP, was checked each morning, and if observed was then defined to be E0.5. Neuronal progenitors were labeled with bromodeoxyuridine (BrdU, Sigma-Aldrich B5002) by intraperitoneal injection (i.p) of $50 \mathrm{mg} / \mathrm{kg}$ BrdU into dams at E8.5-E16, with half-day intervals (with the exception of E14.5 and E15.5). Note, each mouse received only one injection. The concentration was previously validated to label progenitor proliferation in the embryonic brain without inducing obvious toxic effects (Vasistha et al., 2019). Pups were anesthetized by i.p. injection with a combination of xylazine and ketamine on postnatal day (P)7 and perfused transcardially, first with $10 \mathrm{~mL}$ cold PBS and subsequently with $5 \mathrm{~mL}$ cold 4\% PFA.

\section{Brain Fixation and Storage}

For the pig brains, at earlier time points up until E60, the entire fetus was fixed by immersion and the dorsal skull was opened to improve permeation of the fixative. For the later time points, the brains were removed from the skull. Fixation of larger brains was also performed by immersion using 4\% paraformaldehyde (PFA; Millipore Sigma) in PBS (Thermo Fisher Scientific) from $24 \mathrm{~h}$ to up to 2 weeks, dependent on the size of the fetus/brain. Fetuses and brains were then stored long-term at $4^{\circ} \mathrm{C}$ in $0.002 \%$ Sodium Azide (VWR-Bie\&Berntsen) in PBS. For the mouse brains, brains were initially fixed by cardiac perfusion (see section, BrdU labeling). The brains were then removed from the skull and fixed overnight $(\mathrm{O} / \mathrm{N})$ in $4 \% \mathrm{PFA}$ at $4^{\circ} \mathrm{C}$ and stored in PBS with $0.02 \%$ sodium azide.

\section{Paraffin Embedding and Sectioning}

Prior to dehydration, the brains were dissected to approximately $10 \mathrm{~mm}$ thickness. The brains were dehydrated by immersion into a sequential series of ethanol, estisol and liquid paraffin using a tissue Processor (Thermo Fisher Scientific Citadel 2000). Following dehydration, the tissues were embedded in liquid paraffin followed by cooling down on a cold stage. Fivemicrometer $(\mu \mathrm{M})$ thick sections were cut on a microtome (Leica SM2000R) and mounted onto SuperFrost slides (Thermo Fisher Scientific). The sections were dried at room temperature (RT) overnight $(\mathrm{ON})$ and stored long term at $4^{\circ} \mathrm{C}$.

\section{Cresyl Violet Staining}

Paraffin embedded sections were deparaffinized for $45 \mathrm{~min}$ at $60^{\circ} \mathrm{C}$ and $2 \times 10 \mathrm{~min}$ in Xylene. The sections were sequentially rehydrated for $2 \times 5 \mathrm{~min}$ in $99 \% \mathrm{EtOH}, 3 \mathrm{~min}$ in $96 \% \mathrm{EtOH}$, $3 \mathrm{~min}$ in $70 \% \mathrm{EtOH}$, rinsed in tap water and stained for $12 \mathrm{~min}$ in $37^{\circ} \mathrm{C}$ Cresyl Violet (Millipore Sigma). The stained sections were rinsed in distilled water and staining outside of perikarya was differentiated for $10 \mathrm{~min}$ in $96 \% \mathrm{EtOH}$ with $0.02 \%$ glacial acetic acid (Millipore Sigma). The sections were subsequently dehydrated $2 \times 5 \mathrm{~min}$ in $99 \% \mathrm{EtOH}$ and $2 \times 5 \mathrm{~min}$ in Xylene prior to coverslip mounting using DPX (Sigma-Aldrich). All images were acquired on an Axio Scan.Z1 (Zeiss) using automated brightfield imaging.

\section{Tissue-Tek OCT Embedding and Cryosectioning of Porcine Brains}

Fixed fetuses/brains were dehydrated in 30\% sucrose (SigmaAldrich) $/ 1 \times$ PBS (Thermo Fisher Scientific) solution $(0.22 \mu \mathrm{m}$ filtered) at $4^{\circ} \mathrm{C}$ for $48 \mathrm{~h}(\mathrm{~h})$. The brains were cut into small pieces of approximately $4 \mathrm{~mm}$ thickness. The brain tissues were immersed into Tissue-Tek OCT (Sakura) and mounted within plastic molds (Simport) by snap-freezing in N-hexane (VWR) solution immersed within liquid nitrogen. The frozen tissue was stored at $-80^{\circ} \mathrm{C}$ until use. Brain sections were cut at $30 \mu \mathrm{m}$ thickness using a cryostat (Leica CM 1950) and mounted onto SuperFrost Plus slides (Thermo Fisher Scientific) and stored at $-20^{\circ} \mathrm{C}$. 


\section{Cryo-Embedding and Sectioning of Mouse Brains}

Following PFA fixation, mice brains were dehydrated in 30\% sucrose (Sigma-Aldrich) in PBS O/N at $4^{\circ} \mathrm{C}$. One hemisphere was embedded by immersion in O.C.T. Compound (TissueTek, Sakura) in a cryomold, snap-frozen in N-hexane (VWR) submerged in liquid nitrogen, and stored at $-20^{\circ} \mathrm{C} \mathrm{O} / \mathrm{N}$ until used for cryosectioning. The brains were sectioned on a cryostat (Leica CM 1950) at $30 \mu \mathrm{m}$ thickness and mounted onto SuperFrost Plus slides (Thermo Fisher Scientific) and stored at $-20^{\circ} \mathrm{C}$.

\section{Immunohistochemistry}

Paraffin embedded brain sections were deparaffinized for $45 \mathrm{~min}$ at $60^{\circ} \mathrm{C}$ in the oven and $2 \times 10 \mathrm{~min}$ in Xylene (VWR). Subsequently, the sections were sequentially rehydrated for $2 \times$ $5 \mathrm{~min}$ in $99 \% \mathrm{EtOH}, 3 \mathrm{~min}$ in $96 \% \mathrm{EtOH}, 3 \mathrm{~min}$ in $70 \%$ $\mathrm{EtOH}, 3 \mathrm{~min}$ in tap water, and washed $2 \times$ for $5 \mathrm{~min}$ in PBS. The sections were subsequently permeabilized for $30 \mathrm{~min}$ in $0.1 \%$ Triton-X (Millipore Sigma) in PBS at RT and washed $2 \times$ $5 \mathrm{~min}$ in PBS before antigen retrieval in boiling citrate $[0.01 \mathrm{M}$, pH6] (Sigma-Aldrich) for $3 \times 5 \mathrm{~min}$. The sections were washed once for $5 \mathrm{~min}$ in PBS and Lab Vision MultiVision Polymer Detection System (Thermo Fisher Scientific) was used according to manufacturer's protocol with the following specifications: A mix of anti-parvalbumin and anti-calbindin primary antibodies (Supplementary Table 3) were diluted in 1:500 in PBS and incubated at RT for $30 \mathrm{~min}$. The sections were visualized with LVRed and LVBlue.

\section{Immunofluorescence of Porcine Brains}

Cryosectioned brains were air-dried for $1 \mathrm{~h}$ at RT, followed by rehydration in PBS (Thermo Fisher Scientific) for $10 \mathrm{~min}$. After antigen retrieval the sections were permeabilized in $0.25 \%$ Triton X-100/PBS (Sigma-Aldrich) for $10 \mathrm{~min}$ followed by washing in PBS. The brain tissue was blocked in the blocking buffer $[10 \%$ Normal donkey serum (Biowest) 2\% BSA (Sigma-Aldrich)] at RT for $1 \mathrm{~h}$. Sections were incubated with the diluted primary antibody (Supplementary Table 3) $\mathrm{ON}$ at $4^{\circ} \mathrm{C}$ followed by washing in PBS 3 times for 5 min each. The sections were then incubated in diluted secondary antibody for $2 \mathrm{~h}$ at RT and washed in PBS for 3 times for $5 \mathrm{~min}$ each. The sections were counterstained with $10 \mu \mathrm{g} / \mathrm{mL}$ Hoechst 33258 (Sigma-Aldrich) for $10 \mathrm{~min}$ followed by washing in PBS for $5 \mathrm{~min}$ twice. Secondary antibodies were conjugated with either Alexa fluorophores, 488, 594 or 647 and diluted at 1:500 according to the manufacturer's recommendations (Supplementary Table 4). Negative controls were performed by using IgG controls (Supplementary Table 4) and by omitting the primary antibody.

\section{Immunofluorescence of Mouse Brains}

Cryosectioned brains were air-dried at RT for $1 \mathrm{~h}$ and post fixed in $4 \%$ PFA for $10 \mathrm{~min}$. The sections were subsequently washed in PBS for $5 \mathrm{~min}$ and treated with $1 \mathrm{M} \mathrm{HCl}$ for $45 \mathrm{~min}$ at $37^{\circ} \mathrm{C}$. The acid on the slides was neutralized for $10 \mathrm{~min}$ in $10 \mathrm{mM}$ Tris-HCl (Sigma-Aldrich) pH 8.5 and the slides were washed in PBS for $5 \mathrm{~min}$. Antigen retrieval of the slides was performed by submerging the slides into $90^{\circ} \mathrm{C}$ citrate buffer (Sigma-Aldrich) and letting the slides in the solution cool down at RT on the bench for $50 \mathrm{~min}$. For sections co-labeled with BrdUantibody the antigen retrieval step was omitted. The sections were washed $3 \times 5 \mathrm{~min}$ in PBS, permeabilized for $10 \mathrm{~min}$ in $0.25 \%$ Triton $\mathrm{x}-100$ (MERK) in PBS and washed for $5 \mathrm{~min}$ in PBS. Subsequently the sections were incubated with block buffer (10\% normal donkey serum (NDS, Biowest), 2\% BSA (Sigma-Aldrich), $0.2 \%$ Triton $\mathrm{x}-100$ ) for $2 \mathrm{~h}$ and incubated with a combination of primary antibodies against either BrdU/NeuN or SATB2/BCL11B or BrdU/BCL1B/RELN (Supplementary Table 3) diluted in blocking buffer $\mathrm{O} / \mathrm{N}$ at $4^{\circ} \mathrm{C}$, in a dark humidified chamber. The sections were washed $3 \times 5 \mathrm{~min}$ in PBS and incubated with appropriate secondary antibodies conjugated with either Alexa fluorophores, 488, 546, or 647 (Supplementary Table 4) and diluted 1:500 in blocking buffer for $2 \mathrm{~h} \mathrm{RT}$, in a dark humidified chamber. The sections were subsequently washed $3 \times 5 \mathrm{~min}$ in PBS, counterstained for $10 \mathrm{~min}$ with $10 \mu \mathrm{g} / \mathrm{mL}$ Hoechst 33342 (Sigma-Aldrich), washed $3 \times 5$ min in PBS and mounted with a coverslip using buffered glycerol mounting media ( $90 \%$ glycerol (Sigma-Aldrich), $20 \mathrm{mM}$ Tris $\mathrm{pH} 8.0,0.5 \% \mathrm{~N}$-propyl gallate (Sigma-Aldrich).

\section{Image Acquisition}

For acquisition of Cresyl Violet images, a bright-field microscope, Leica DMR with camera Leica DFC490 and Zeiss Axio scan.Z1 were used to capture the images. For acquisition of immunohistochemistry images, a confocal microscope Leica TCS SPE was used. Acquisition was performed with a $40 \times / 1.15$ oil immersion objective. Confocal images were acquired using Leica LAS X. Images were optimized for brightness and contrast using Fiji-ImageJ. Z-stack images were acquired and used for quantification and afterward overlapped in Fiji-Image J using the function ZProjection with the projection type Max Intensity for visualization. Brightness and contrast were optimized in FijiImageJ post-acquisition. Images spanning the entire width of the EC were acquired as a series of overlapping images and sequentially stitched together post-acquisition using the FijiImageJ Pairwise stitching function. To evaluate the number of OLIG2 ${ }^{+}$cells in the MEC, we captured $z$-stack images from 3 sections from each developmental stage of either the right or left hemisphere. A range of 489-2315 cells were counted for each section, with variation depending on the age of the specimen. To evaluate the $\mathrm{VZ}$, we evaluated 3 sections from each age to assess the number of $\mathrm{EOMES}^{+} / \mathrm{PAX}^{+}$and $\mathrm{EOMES}^{+} / \mathrm{PAX} 6-$ cells (Supplementary Table 1). In the case of $\mathrm{BrdU}^{+}$cells we captured images of $8 \mathrm{z}$-stacks of one section from the right hemisphere of the brains from three different pups of the same litter for each time point (Supplementary Table 2). The immunofluorescent images were acquired on a Leica TCS SPE confocal microscope using LAS X software (Leica). The same settings were used across samples from the experiment.

\section{Structural MRI}

A 9.4T BioSpec 94/30 USR spectrometer (Bruker BioSpin, Ettlingen, Germany) equipped with a $240 \mathrm{mT} / \mathrm{m}$ gradient system 
was used to acquire anatomical images of postmortem porcine brains (E60, E70, E80, E100, P75). Prior to imaging, the PFA fixed brain were suspended into plastic containers filled with a proton-free perfluorinated susceptibility-matching fluid (Solvay Galden HT-230). We used a 35-mm (for P75) and a 23-mm (for E60, E70, E80, and E100) inner diameter transmit/receive volume coil (Bruker). The MR system was interfaced to a console running ParaVision software 6.0.1 (Bruker BioSpin). The parameters used in the brain scans were optimized for gray/white matter contrast: T2-weighted $2 \mathrm{D}$ rapid acquisition with relaxation enhancement (RARE) pulse sequence with $\mathrm{TR} / \mathrm{TE}=20000 / 40 \mathrm{~ms}$ (before birth), 12000/60 ms (after birth), Rare factor $=8$ (before birth), 16 (after birth), in-plane resolution $=100 \mu \mathrm{m} \times 100 \mu \mathrm{m}$, slice thickness $=200 \mu \mathrm{m}$ (before birth), $500 \mu \mathrm{m}$ (after birth). 3D fast low angle shot (3DFLASH) pulse sequence with TR/TE $=30 / 4.6 \mathrm{~ms}$, Flip angle $=10$, $\mathrm{NA}=16$, spatial resolution $=47 \mu \mathrm{m} \times 47 \mu \mathrm{m} \times 70 \mu \mathrm{m}$. The total imaging time was $2 \mathrm{~h}$.

\section{Diffusion MRI Tractography}

Ex vivo diffusion tensor imaging (DTI) of P75 brain $(n=3)$ was obtained using a Stejskal-Tanner sequence $(\mathrm{TR} / \mathrm{TE}=3500 / 17.5$ $\mathrm{ms}, \mathrm{NA}=2$, spatial resolution $=390 \mu \mathrm{m} \times 390 \mu \mathrm{m} \times 390 \mu \mathrm{m})$ on a $9.4 \mathrm{~T}$ spectrometer equipped with a $1500 \mathrm{mT} / \mathrm{m}$ gradient system and a 40-mm inner diameter transmit/receive volume coil. We applied the motion probing gradients (MPGs) in 60 noncollinear directions with $\mathrm{b}=2500 \mathrm{~s} / \mathrm{mm}^{2}$ in addition to $4 \mathrm{~b} 0$. Diffusion Toolkit (version 0.6.4.1) and TrackVis (version 0.6.1) were used for 3D reconstruction of white matter tracts. We used a DWI mask threshold and an angular threshold of 45 degrees. Two regions of interest (ROIs) were selected a priori for the DTI analysis: including the LEC and MEC. ROIs were created by manually segmentation with ITK-snap (version 3.6.0, Yushkevich et al., 2006). The fiber tracks obtained from the ROIs were qualitatively analyzed for the direction of tracks, and differences in FA values within the brain parenchyma. The assessment was done using tractography maps: (1) a standard color-coded reconstruction to visualize the location and orientation of white matter pathways (blue tracks represented the axonal orientations in the craniocaudal, green in anterior-posterior, and red in left-right direction); (2) a scalar FA map with minimum and maximum FA thresholds of 0.1 and 0.6 , respectively, and (3) total track length information for each ROI.

\section{Statistics and Quantification}

For immunohistochemistry and assessment of neuroconnectivity from DTI white matter tracts, all samples were performed in biological triplicates (three different brains). Statistical analysis was conducted using commercially available software- Prism 7.0 (GraphPad Software). Dependent on the experimental design, an ordinary one-way ANOVA or an unpaired two-tailed $t$-test was performed to statistically assess differences. All error bars denote standard deviation (SD). Non-significant $p$-value (n.s.p) $>0.05$. Significant differences are marked in figures using asterixes which represent the following: ${ }^{*} P \leq 0.05,{ }^{* *} P \leq 0.01,{ }^{* * *} P \leq 0.001$.
The OPCs were counted in across the defined anatomical layers and quantified using the grid and cell counting function of the ImageJ-NHI software.

The counting of NeuN and BrdU immunolabeled cells in the mouse brain sections was performed manually using the Cell Counter tool in Fiji. The cell counting was performed on each individual of the $8 \mathrm{z}$-plane images that were later merged, to ensure that overlapping cells were counted only once. Anti-BrdU labeled cells were only counted as positive if a strong labeling was observed (at least $50 \%$ staining). The quantity of NeuN and BrdU positive cells were counted in the layer (L)II, LIII and LV / VI of the MEC in sections from three separate brains with BrdU injection at each time point. For the remaining stages of BrdU injection only two separate brains were quantified. The fraction of neurons with BrdU incorporation was calculated as the fraction of BrdU positive/NeuN positive cells. The mean and SD of the fraction of BrdU positive neurons was calculated in $\mathrm{R}$ for the different layers at each individual stage of BrdU injection. The $\mathrm{SD}$ was calculated in $\mathrm{R}$, using the $\mathrm{sd}($ ) function. The number of counted cells in each layer in each brain section at the different timepoints is reported in Supplementary Table 5.

\section{RESULTS}

\section{The MEC and LEC Boundaries Can Be Delineated in the Prenatal Porcine Brain}

The pig is not traditionally used as a model to study the EC or brain development, hence, it was important to validate that the pig EC was highly similar to other species. Gestational age differences and trimester lengths were first compared between mouse, man and pig to determine when corticogenesis occurs in the pig. The transition from trimester 1 to 2 is an important early time point, since this is when neural tube formation and gastrulation is completed in mouse and man (Patten et al., 2014). Other key developmental features, such as fusion of the palate also mark this transition, as well as gonadal differentiation. Gonadal differentiation has been studied in the pig and occurs at $\mathrm{E}$ (embryonic day) 50 in the pig (Pontelo et al., 2018). We therefore deduced that the end of the first trimester occurs at E50. The transition from trimester 2 to 3 marks a significant and continual increase in human fetal weight upon completion of organogenesis. A similar and marked increase in fetal weight occurs at E70 in the pig (Kim, 2010). We believe, therefore, that E70 marks the transition from trimester 2 to 3 . We highlight this comparative overview of trimester lengths between species in Figure 1A.

We then performed a detailed characterization of the location of the EC in a late gestational pig brain at E100 (2 weeks prior to birth). In the pig, the EC is located in the piriform lobe, caudal to the olfactory (piriform) cortex and the cortical nucleus of amygdala (COA) and hippocampal transition area (HA) (Figure 1B). Cresyl violet staining revealed a cytoarchitecturally mature EC, with similar morphology as the postnatal EC (Figure 1C and Supplementary Figure 1). Layer IV was distinctively acellular, which is a typical feature in the adult EC known as the lamina dissecans. We identified the parasubiculum 

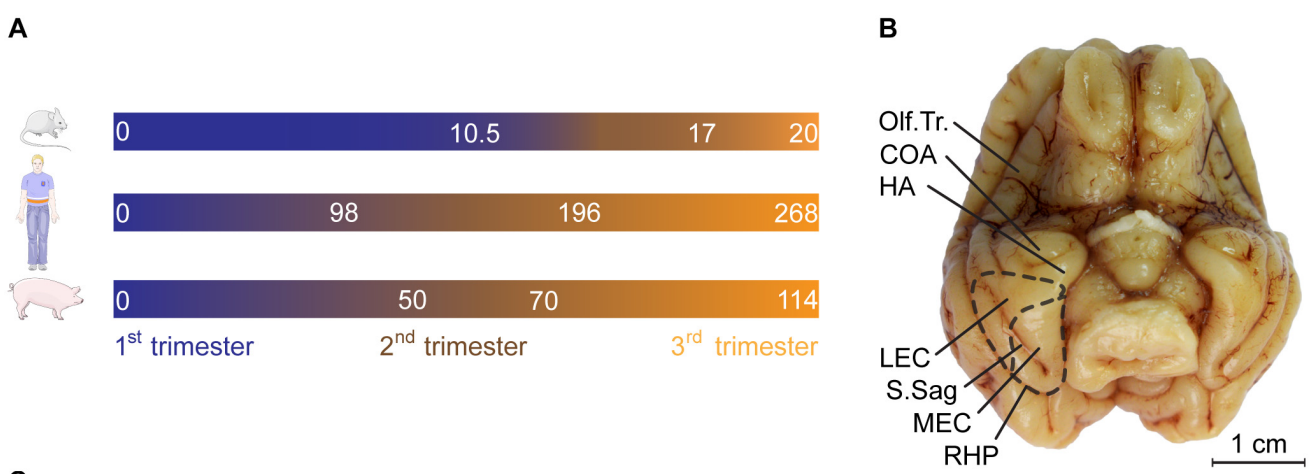

C
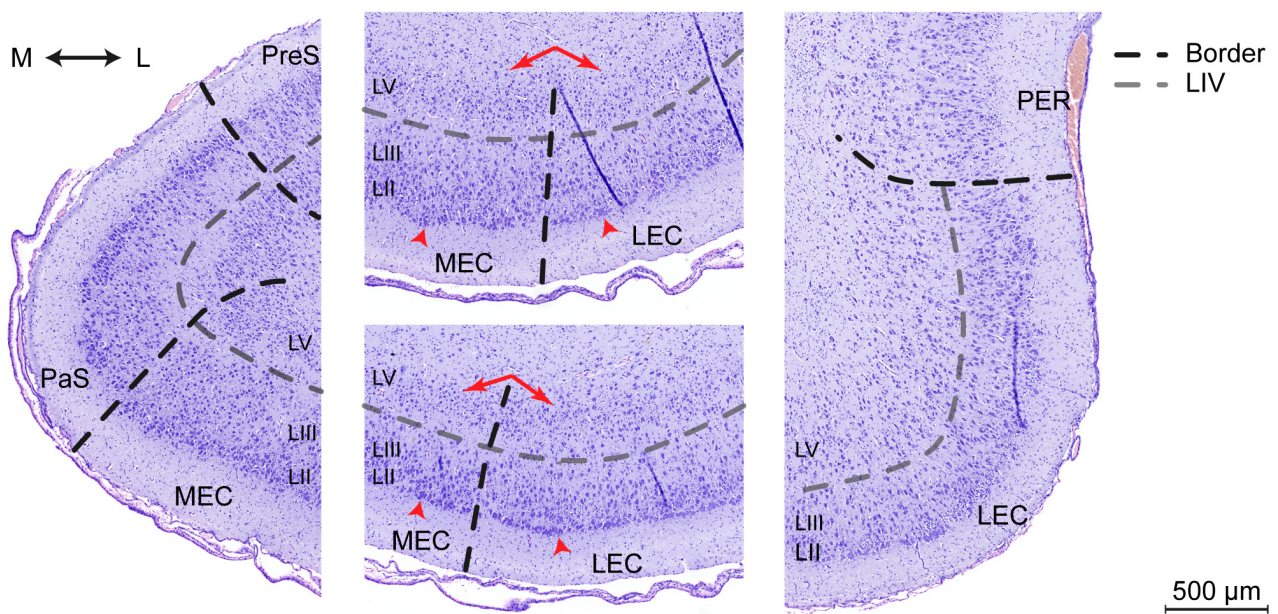

D

E100

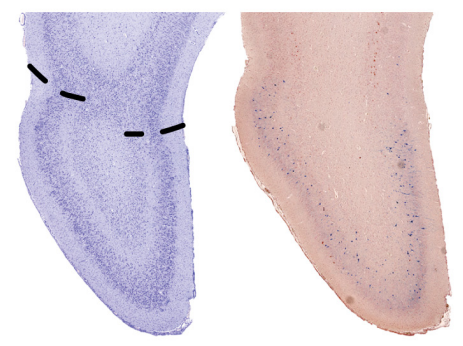

Cresyl violet

Parvalbumin / Calbindin

MEC border - -

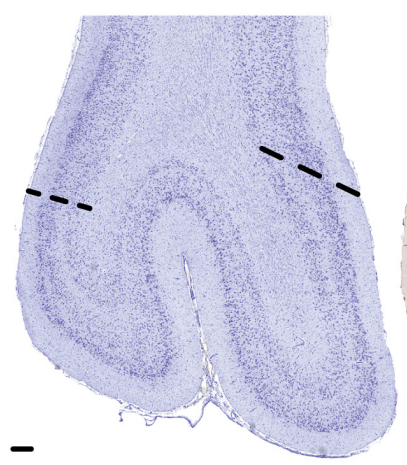

P75

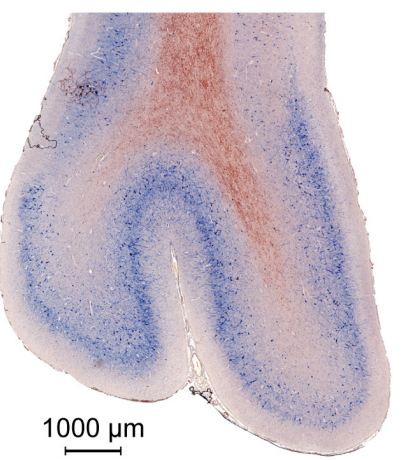

FIGURE 1 | Cytoarchitectonic features of the developing porcine entorhinal cortex. (A) Length and trimester divisions of the gestation in mouse, human, and pig. (B) Macroscopic view of the ventral surface of the porcine brain at Embryonic day (E)100 with annotation of the piriform lobe including the entorhinal area. Dashed black line indicates the outline of EC and the border between lateral and medial entorhinal cortex. Olfactory tract (Olf.Tr.); caudate nucleus of amygdala (COA); hippocampal area (HA); lateral entorhinal cortex (LEC); medial entorhinal cortex (MEC); sagittal sulcus (S.Sag); posterior rhinal sulcus (RHP; note that the sulcus coincides with the visible lateral border of the EC). Scale bar $1 \mathrm{~cm}$. (C) Delineations and cytoarchitectonic features of cresyl violet stained coronal sections of the EC (shown at E100 as representative). Light gray punctuated line marks the acellular layer (L)IV lamina dissecans, black punctuated line borders between MEC and LEC or EC and adjacent areas. Red arrows (middle panels) shows the difference in the cellular organization in deep layers, arrowheads highlight large superficial cells of the EC. Medial (M); lateral (L); pre-subiculum (PreS); para-subiculum (PaS), perirhinal cortex (PER). Scale bar $500 \mu \mathrm{m}$ (D) Cresyl violet staining and parvalbumin (PVALB) expression highlights the caudal boundaries of the EC at E100 and P75 (coronal plane). PVALB expression coincides with defined cytoarchitectonic borders of the EC. Scale bar $1000 \mu \mathrm{m}$.

(PaS) medial to the EC. The PaS could be identified at E100 from its similar cytoarchitecture as the EC. One exception was that the soma sizes of the PaS LII and LIII cells were more equal, compared to the EC. In the EC, the LII cells have somas approximately twice as large as in the LIII (Witter et al., 2017). We could identify the perirhinal cortex (PER) located directly adjacent and lateral to the EC through the disappearance of large superficial cells of the LII typical of the EC, together with 
the disappearance of the lamina dissecans (Figure 1C). Already at E100, the EC can be easily divided into a lateral (LEC) and medial (MEC) subdivision. This is based on cytoarchitecture differences described in other species such as rodents, dogs, cats and humans (Wyss et al., 1983; Insausti et al., 1995; Woznicka et al., 2006; Gatome et al., 2010). The pig MEC presented deeper layers structured with a narrow homogenous and densely packed cellular layer organized with a columnar appearance. In the transition into the LEC, the deep layers widened with a lower cell density and a disordered cell deposition. The LIV lamina dissecans in the MEC was distinct and acellular, while in the LEC, the lamina dissecans was more diffuse, with more cellular infiltration (Figure 1C). The MEC gradually occupied the entire mediolateral entity along the rostrocaudal axis of the piriform lobe. The MEC caudally borders to the neocortex as the piriform lobe disappears. This boundary was traced by immunolabeling for parvalbumin (PVALB) positive interneurons (Figure 1D), as seen in the rat (Wouterlood et al., 1995). We also traced the immunoreactivity of Calbindin (CALB), which in the rat has proven useful to delineate the EC (Boccara et al., 2015), however, expression was not observed in EC neurons (Figure 1D). We observed a sulcus forming at the mid-rostrocaudal extent of the EC, persisting to the caudal end of the piriform lobe which was also visible macroscopically (Figure 1B). This sulcus, known as the sagittal sulcus (S.Sag) has been previously reported in the domestic (Holm and Geneser, 1989) and Göttingen minipig (Bjarkam et al., 2017), but not in other gyrencephalic species, such as dogs, cats, and humans (Wyss et al., 1983; Insausti et al., 1995; Woznicka et al., 2006). We observed the S.Sag to coincide with the appearance with the MEC on the rostrocaudal axis (Supplementary Figure 1), which provided a unique surface landmark for microscopically and macroscopically assessing the MEC and LEC borders. This anatomical study was important for assessing the borders of the MEC for further detailed investigation.

\section{Prominent Oligodendrocyte Precursor Cells Are a Major Anatomical Feature in the Superficial Layers of the Developing Porcine LEC and Perirhinal Cortex}

Interestingly, we observed a prominent number of oligodendroglia-like cells present in the superficial layers of the LEC and in the adjacent perirhinal cortex (PER), which has not been previously highlighted in other anatomical descriptions of the EC. These cells were proximal and clustered around large entorhinal cells (Figure 2A). Morphologically, these appeared to be oligodendrocyte precursor cells (OPCs) with clear Nissl free cytoplasm and a large round Nissl stained nucleus (GarciaCabezas et al., 2016; Figure 2B). We observed an enrichment of these oligodendroglia-like cells in the superficial layers of the LEC and PER from E80 of development and fewer cells were observed in the MEC and cingulate gyrus (dorsal neocortex) (Figure 2B). In the most lateral part of the LEC, these OPClike cells were evenly distributed within the superficial layer but converged into distinct islets toward the MEC. The islets disappeared at the border of the LEC-MEC and the OPC-like cells were more evenly distributed within the MEC. We were not able to distinguish these cells using cresyl violet staining prior to E80 (Supplementary Figure 2), therefore we immunolabeled the MEC from E50 onwards with the OPC marker, OLIG2 in order to determine if they may be present earlier. We detected $\mathrm{OPC}^{+}$cells from E50 onwards (Figure 2C). At E50, the OLIG2 ${ }^{+}$ cells were detected predominantly in the subplate and from E60 onwards were distributed more evenly across the developing cortical layers (Figure 2D).

\section{The Developing EC Accelerates in Growth at the End of the Second Trimester}

We subsequently analyzed the developmental timing of the EC using cresyl violet staining (E50-P75) and structural postmortem MRI (E60-P75). No features of an EC could be determined prior to E50. At E50, we identified prominent and large soma of entorhinal-like cells in the superficial layer of the ventral telencephalon before any distinct layer was formed (Figure 3A). The three-layered cortex became six-layered by E60 and the lamina dissecans was now visible (Figure 3B). At E60, we were able to delineate the MEC from the LEC based on the deposition of the deep layer cells and the higher cellularity of the lamina dissecans in the LEC. Together, this indicated that the EC cortical layers form during the second trimester between E50 and E60 in the pig. At E70 (late in the second trimester), we observed the S.Sag formation microscopically and cells in the superficial LII were prominent, large and darkly stained nuclei with Nissl (Figure 3C). From E80, the large cells in LII appeared more prominent (Figure 3D) and the S.Sag was macroscopically visible (Figure 3E).

The positional anatomy and rate of EC growth was evaluated using postmortem structural-MRI. The EC was annotated from E60 to P75 based on our histological descriptions and macroscopic features (Figure 4A). We calculated the volume of the EC and the remaining cortex based on the MRI annotations and found the EC growth was linear from E60 to P75 (Figure 4B). When we compared the growth rate of the EC to that of the whole cortex of the brain, we found that the EC had a significantly higher growth rate at E70 during the late second trimester (Figure 4B), suggesting a local and specific developmental growth period at this time point. We were unable to detect whether the growth was due to expansion of the white or gray matter, due to the lack of MRI resolution. In summary, we identified a significant growth spurt during the late second trimester attributable to either gray and/or white matter expansion.

\section{Neuronal Projections From the Postnatal EC Reach Several Brain Regions}

We then analyzed the EC and its connectivity to other brain regions using DTI tractography in a later and more developed EC, in postnatal (P) brains 75 days after birth (P75). A high number of white matter tracts emerged from both the MEC [mean 6031 \pm 1319; standard deviation (SD)] and the LEC [mean $3329 \pm 4099$ (SD)] (Figures 5A,B and Supplementary Table 6). 
A
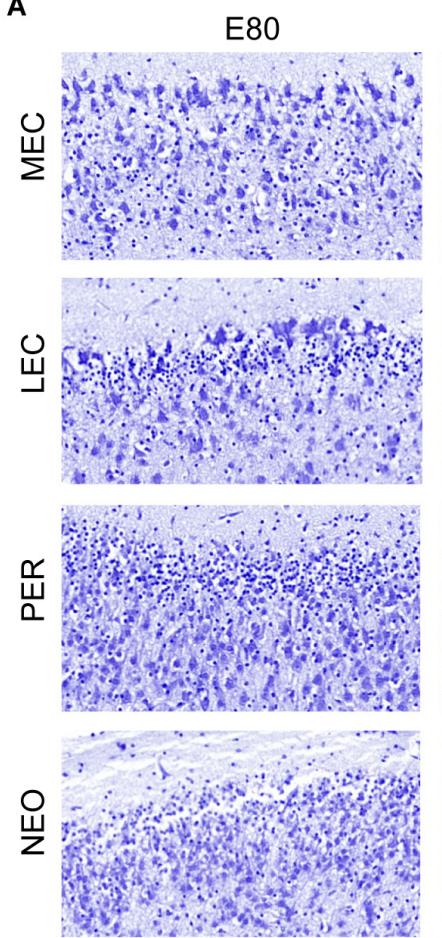

E100
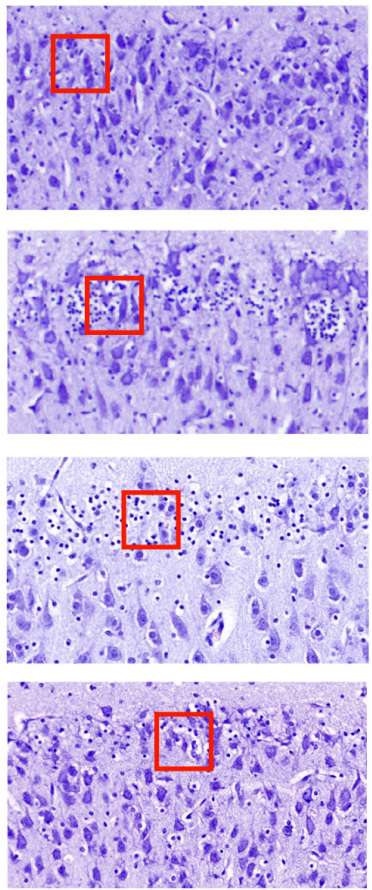

P75
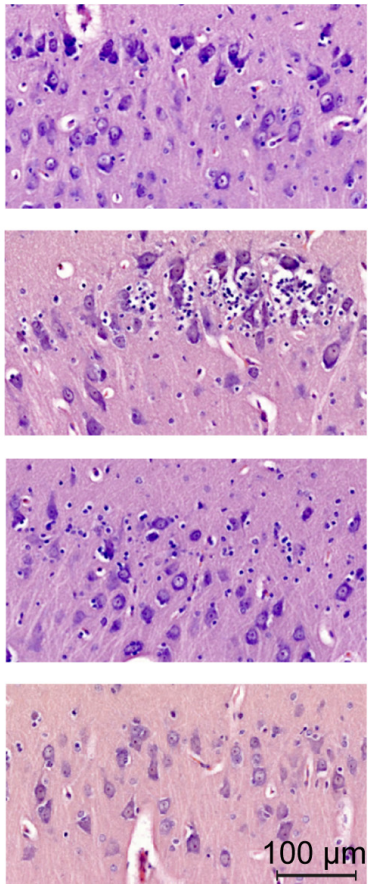

B
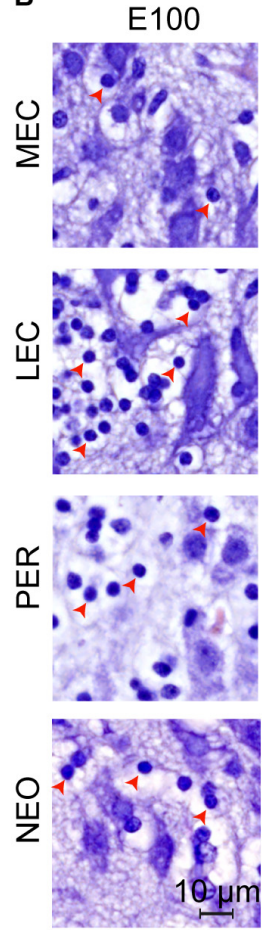

P75

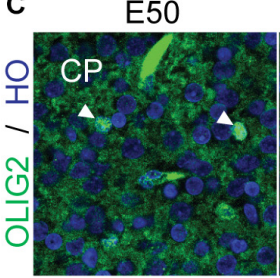

E60

E70

E80
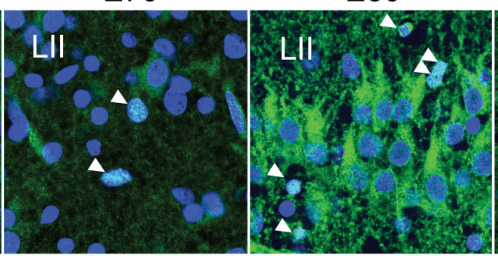

E100

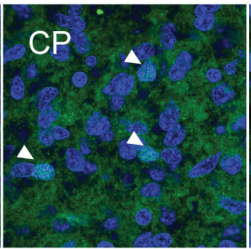

E60
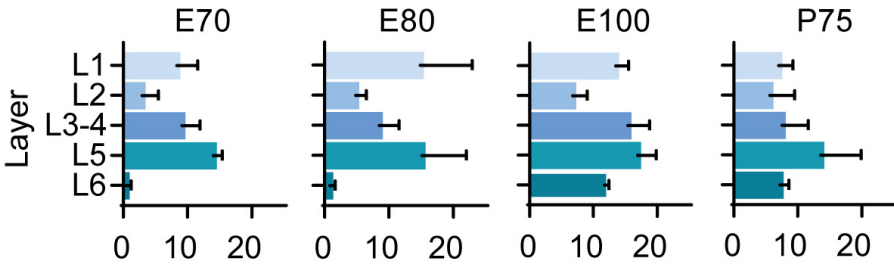

Percentage of OLIG2+ cells in each layer/zone (\%)

FIGURE 2 | Oligodendrocyte precursor cells (OPCs) emerge in the entorhinal cortex (EC) early in the second trimester. (A) A high number of OPCs are present in the superficial layers of the lateral entorhinal cortex (LEC) and perhirhinal cortex (PER) and less OPCs observed in the medial entorhinal cortex (MEC) and neocortex (NEO) at different Embryonic days (E) and at Postnatal day (P) 75. Scale bar $100 \mu \mathrm{m}$. (B) High magnification of red squares in (A) depicts the morphology of the OPCs at E100 which shows condensed nuclei and nissl free cytoplasm (red arrowheads). Scale bar $10 \mu \mathrm{m}$. (C) OLIG2 expression and Hoescht (HO) staining in the developing EC. Images taken within the developing cortical plate (CP) (E50-E60) and later (E70-P75), in Layer II (LII). Scale bar 25 $\mu$ m. (D) Quantification of OLIG2 positive cells in the EC by zones/cortical layers from E50 until P75. Cortical plate, CP; intermediate zone, IZ; marginal zone, MZ; subplate, SP; subventricular zone, SVZ ventricular zone, VZ. All error bars represent SD.

Projections extended to the peri- and post-rhinal cortex, the hippocampus, hippocampal commissure, olfactory bulb, amygdala, nucleus of the lateral olfactory tract, putamen and nucleus accumbens (Figures 5C-H, Supplementary Video 1, and Supplementary Table 1). Further connections between the MEC and LEC could also be observed (Supplementary
Table 1 and Supplementary Video 1). The MEC connected to more regions than the LEC (Figure 5H) and most connections were observed to the amygdala and subiculum (Figure $5 \mathbf{H}$ and Supplementary Table 1). Most of the LEC connections traced also to the amygdala (Figure 5H and Supplementary Table 1). Regarding connectivity to the hippocampus, both the LEC and 


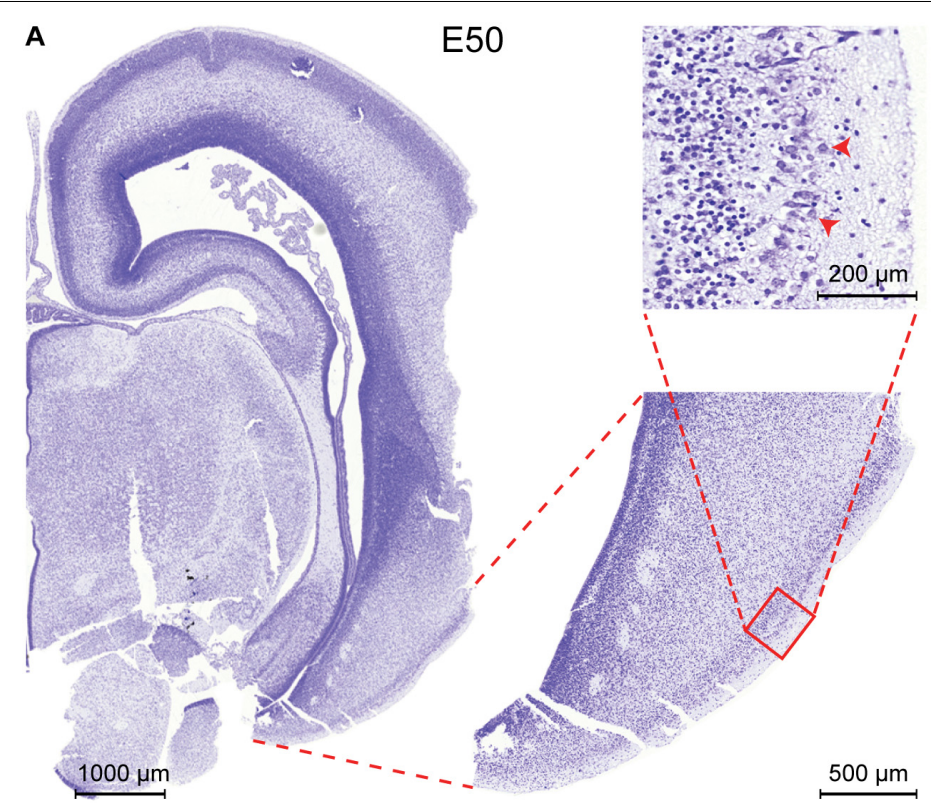

C

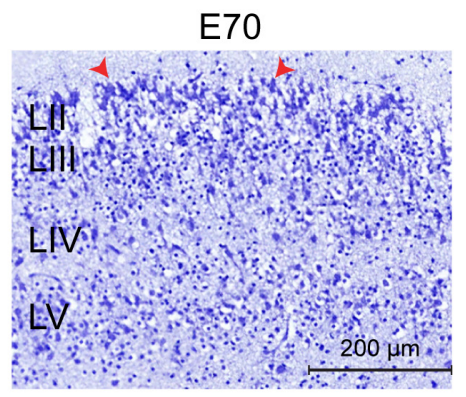

D

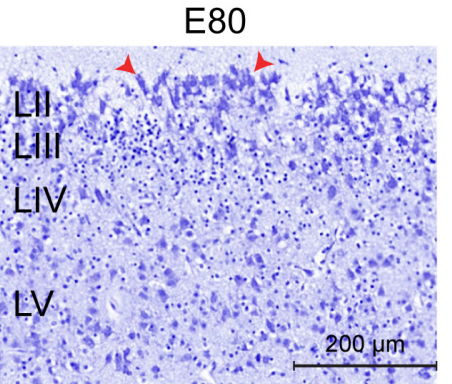

B

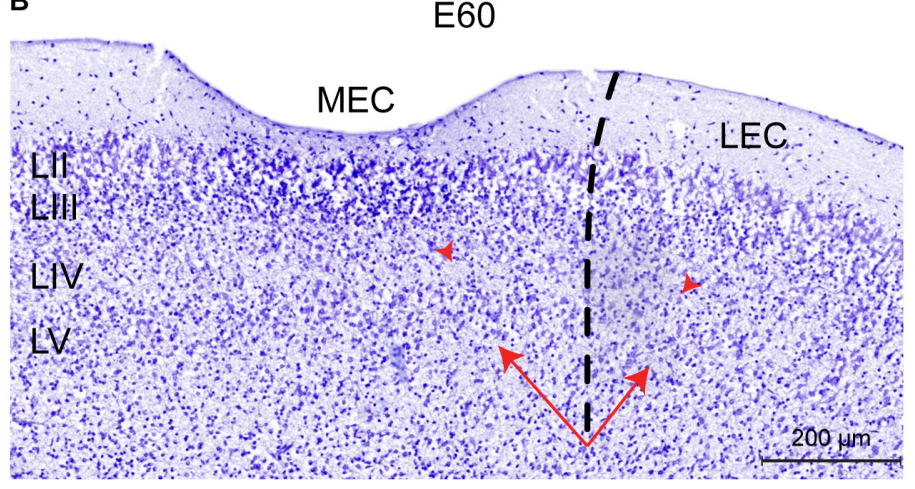

E

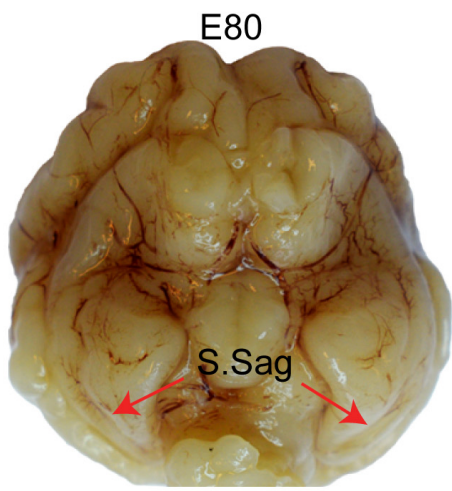

FIGURE 3 | The entorhinal cortex (EC) forms between Embryonic day (E)50 and E60 in the pig. (A) Entorhinal-like cells with large somas (red arrowheads) are identifiable at E50 in the superficial layer of the ventral telencephalon. Scale bars $1000 \mu \mathrm{m}, 500 \mu \mathrm{m}$, and $200 \mu \mathrm{m}$. (B) At E60 the EC becomes six-layered, the lamina dissecans becomes apparent as layer (L)VI, and the medial EC (MEC) and lateral EC (LEC) subfields of the EC become distinguishable, based on the difference in cellularity of the lamina dissecans (red arrowheads) and the deep layer (LV-LVI) organization (arrows). Scale bar $200 \mu \mathrm{m}$. (C) Section of the MEC at E70. Prominent, large and darkly stained nuclei in the superficial LII (arrowheads) are apparent. Scale bar $200 \mu \mathrm{m}$. (D) Section of the MEC at E80. Superficial LII cells become more prominent. Scale bar $200 \mu \mathrm{m}$. (E) The sagittal sulcus (S.Sag) becomes macroscopically visible in the piriform lobe at E80 (red arrows).

MEC connected to the DG, CA1, and CA3 regions, although more connections were captured from the MEC (Figure $\mathbf{5 H}$ and Supplementary Table 1). A small number of fibers that emerged from the MEC projected to the dorsal hippocampal commissure (Figure 5G and Supplementary Table 1). Strikingly, most of the tracts traced to other regions with a large number extending to the rostroventral brain, presumed to be the orbitofrontal cortex or potentially the anterior visual cortex (which remains unannotated in the pig). Many LEC fibers ending at the rostral end of the brain, ended in a slightly caudal (unannotated) location to the MEC fibers (Supplementary Video 1). To sum, the connectivity between the EC and septohippocampal region in the postnatal pig brain was remarkably similar to that which has previously been reported in other species, including humans
(Wilhite et al., 1986; Witter et al., 1986, 2017; Mufson et al., 1990; Totterdell and Meredith, 1997; Kolenkiewicz et al., 2009; Rowland et al., 2013; Sun et al., 2014).

\section{The Developing MEC Is Marked by Parallel Lamination}

Given evidence suggests the layers of the EC may not form or mature in the classic inside-out lamination pattern, we decided to trace the cortical lamination patterning and focused on the MEC where the stellate cells reside and are known to drive maturation events (Donato et al., 2017). The timing of neurogenesis was investigated by performing immunohistochemistry at 8 time points from E23 to P75 of development. Using antibodies 

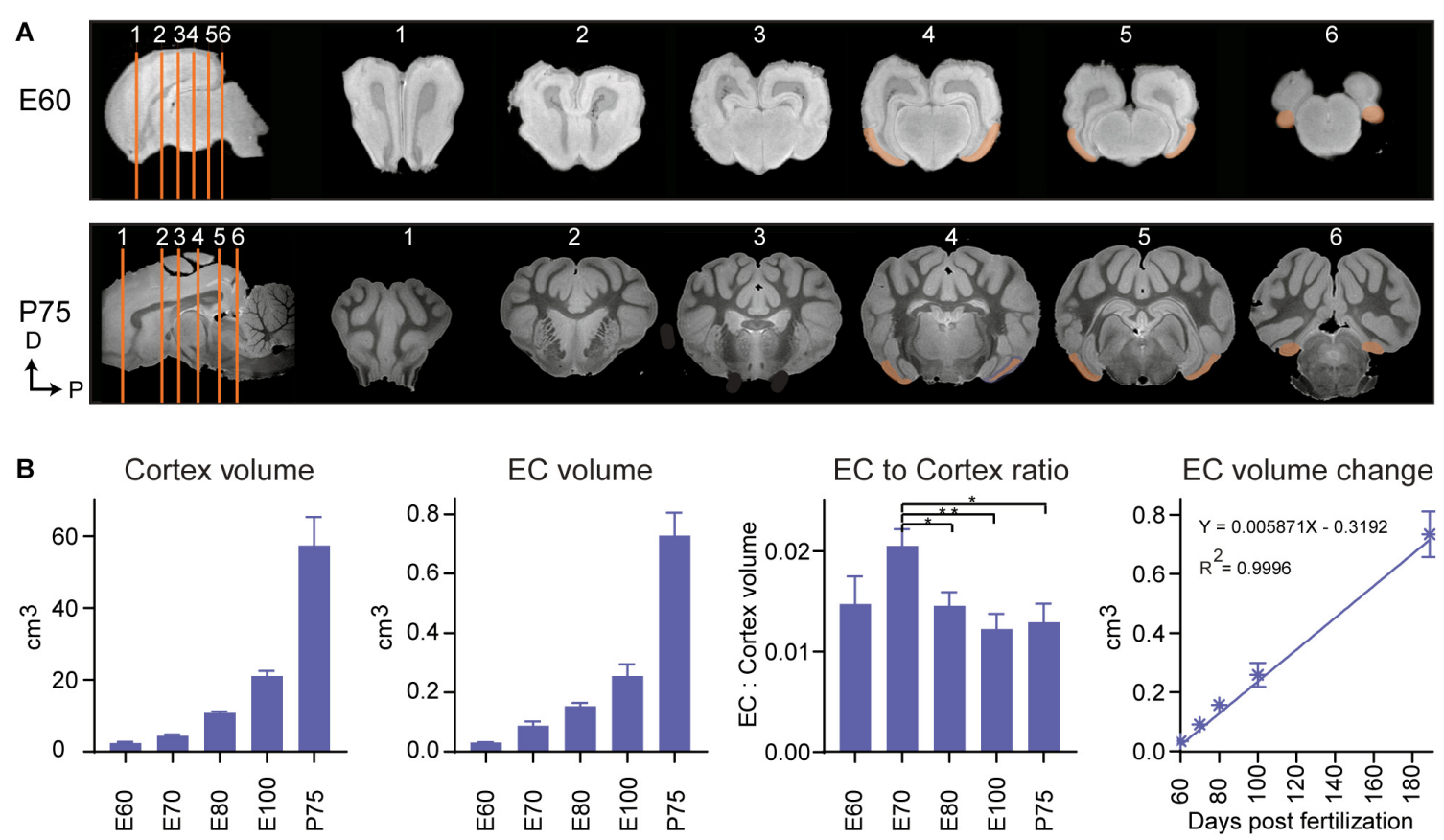

FIGURE 4 | Magnetic resonance imaging (MRI) of the developing entorhinal cortex (EC) shows extensive growth at the end of the 2nd trimester. (A) MRI shows the rostral to caudal position of the early developing EC at embryonic day (E)60 and postnatal day (P)75 in the coronal plane (sections depicted by orange lines). Dorsal (D) and Posterior (P). (B) The total cortex volume, the EC volume, the EC:Cortex ratio and the change in EC volume over time shows an overall linear growth pattern during development, but an exceptional growth spurt of the EC compared to the rest of the neocortex at E70 during development.

directed against GFAP, FABP7 (BLBP), SOX2 and PAX6, we could detect the neural epithelium (NE) $\left(\mathrm{PAX}^{+} / \mathrm{FABP}^{-}\right)$at E23 and radial glia $\left(\mathrm{GFAP}^{+} / \mathrm{FABP}^{+}\right)$at E26 (Supplementary Figures $3 \mathbf{A}-\mathbf{C})$. We measured the size of the ventricular zone (VZ) $\left(\mathrm{SOX}^{+} / \mathrm{PAX}^{+}\right)$and identified that it was present at E23 and peaked in size at E50 (Supplementary Figure 3D). After this time point, the $\mathrm{VZ}$ declined dramatically in size at E60 (Supplementary Figure 3D). We then investigated the second germinal zone by performing immunohistochemistry using antibodies directed against EOMES and PAX6. Our results showed the presence of a subventricular zone (SVZ) containing different populations of EOMES/PAX6 progenitors (Supplementary Figures 3E,F). Later in neurogenesis the SVZ splits into an inner- and outer- subventricular zone (ISVZ and OSVZ), with the OSVZ being much larger in size in humans and non-human primates, compared to ferrets and rats (Smart et al., 2002; Fietz et al., 2010; MartinezCerdeno et al., 2012). Assessment and quantification of the porcine SVZ at E26 showed that $\mathrm{EOMES}^{+} / \mathrm{PAX}^{+}$cells and EOMES $^{+} / \mathrm{PAX} 6$ - cells were present at an equal ratio (Supplementary Figure 3F). At E33, two distinct layers could be observed above the VZ, a dense inner subventricular zone (ISVZ) containing equal proportions of $\mathrm{EOMES}^{+} / \mathrm{PAX}^{+}$ and EOMES $^{+} / \mathrm{PAX} 6(-$ cells and a diffuse overlying OSVZ containing EOMES(+/PAX6(+ and EOMES(+/PAX6(- cells (Supplementary Figures S3E,-F). We observed that the ratio between $\mathrm{EOMES}^{+} / \mathrm{PAX}^{+}$and $\mathrm{EOMES}^{+} / \mathrm{PAX}^{-}$cells changed over time, with $\mathrm{EOMES}^{+} / \mathrm{PAX}^{+}$cells becoming more abundant in both the ISVZ and OSVZ (Supplementary Figure 3F). These zones had declined by E70. Considering PAX6 is an important transcriptional regulator of neurogenesis (Sansom et al., 2009) and Eomes is specifically expressed in neural progenitors (Arnold et al., 2008), our findings imply that neurogenesis within the porcine EC starts after E28 and before E33, continuing until shortly after E70.

We then analyzed the events of cortical lamination in the EC by using immunohistochemical analyses of canonical markers for deep (BCL11B) and superficial layers (SATB2), as well as EOMES, RELN and TBR1 across varying times of gestation in the pig. During early cortical formation (E21), we detected the presence of $\mathrm{EOMES}^{+}$and $\mathrm{RELN}^{+}$cells toward the pial surface (Supplementary Figure 3G). Co-expression of EOMES $^{+} / \mathrm{RELN}^{+}$cells were detected 2 days later at E23 and expression of TBR1 emerged in some of these cells at E23 with more $\mathrm{RELN}^{+} / \mathrm{TBR}^{+}$cells detectable by E33 (Supplementary Figures $3 G, H$ ) which we deduced to be migrating and maturing Cajal-Retzius cells (CR cells) that are important for regulating cortical lamination (Hevner et al., 2003). We then traced local neurons using BCL11B and SATB2. As expected, BCL11B was the first of the two markers to be expressed at E33 and was located in the cortical plate (Supplementary Figure 4). Interestingly, a large proportion of the $\mathrm{BCL}_{11} \mathrm{~B}^{+}$cells expressing SATB2 in the cytoplasm could be detected in the main cortical layer at E39 (Supplementary Figure 4). By E50 a distinct new population of BCL11B cells had emerged as a superficial layer of the EC which could not be observed in the dorsal telencephalon 

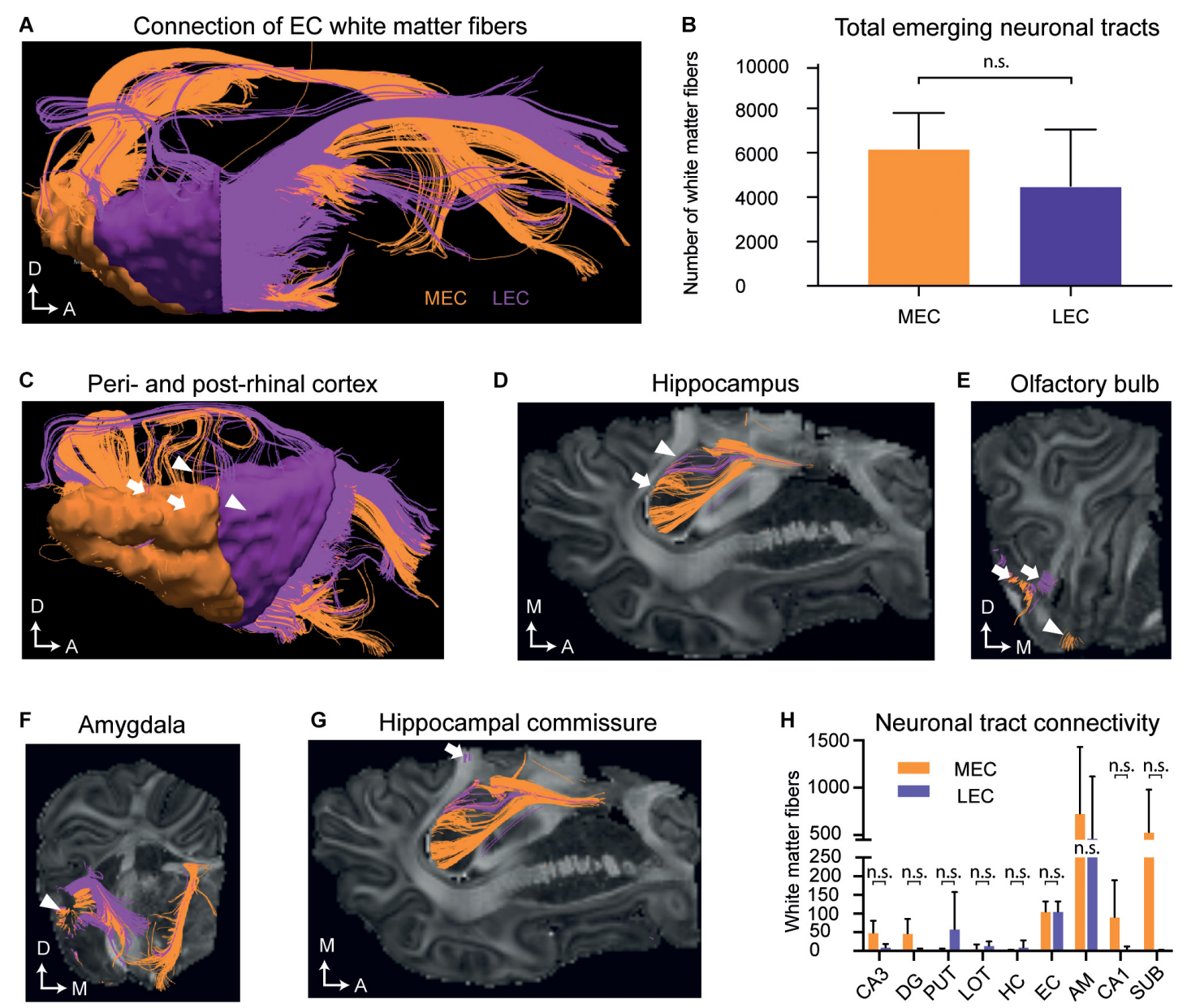

FIGURE 5 | Diffusion tensor imaging (DTI) of the white matter projections identify white matter fiber projections to varying brain regions at postnatal day (P)75. (A) An overview of white matter fibers connecting to/from the medial entorhinal cortex MEC (orange) and laternal entorhinal cortex LEC (purple). (B) The average number of tracts connecting to/from the MEC and LEC do not differ significantly. (C) White matter fibers connecting to/from the perirhinal cortex (white triangles) and postrhinal cortex (white arrows). (D) White matter fibers connecting to the hippocampus (white arrows). (E) White matter fibers connecting to the lateral (white arrows) and medial olfactory bulb (white triangle). (F) White matter fibers connecting to the amygdala (white triangle). (G) White matter fibers extend from the EC to the hippocampal commissure (white arrow). Axes: A, Anterior; D, Dorsal, M, Medial. (H) The average number of tracts connecting to different anatomical regions emerging from the EC do not significantly differ between the MEC and LEC. All error bars denote SD. n.s, not significant. Significance threshold, $p \leq 0.05$. AM, amygdala; DG, dentate gyrus; EC, entorhinal cortex; HC, Hippocampal commissure; LOT, lateral olfactory tract; PUT, putamen; SUB, subiculum.

(Figures 6A,B). These large prominent nuclei resembled the prominent large entorhinal cells that were observed histologically (Figure 1C). In the dorsal telencephalon, a SATB2 ${ }^{+}$population emerged in the superficial layer at E50 which remained present until gestation (Figures 6A,B) which was not detected in the EC. In contrast, a new population of BCL11B+ cells emerged at E60 underneath the superficial layer of $\mathrm{BCL}_{11 \mathrm{~B}}{ }^{+}$cells. This indicated that the porcine EC was forming in a non-conventional pattern. This cell layer positioning was retained even after birth (Figure 6C) and was not observed in the dorsal telencephalon (Figure 6B). A small proportion of the LII $\mathrm{BCL}_{11 \mathrm{~B}}^{+}$cells began to express RELN at E60 (10 days after deposition) with expression becoming more abundant in the superficial cells at E70 (Figures 6B,C). The $\mathrm{RELN}^{+}$neurons residing in both the MEC and LEC continued to express BCL11B and SATB2 after birth (Figures 6D,E).
An independent approach that would support our observation of non-conventional pattern of MEC formation would be to study the timing of cortical layer deposition using bromodeoxyuridine (BrdU) labeling. Since the quantities of BrdU required for administration of $>200 \mathrm{~kg}$ pregnant sows were not feasible, we selected the mouse as a translational model. Injections were performed at half day intervals from E8.5 to E14 and 1 day intervals from E14 to E16 to capture the entire span of neurogenesis. Brains were collected from P7 mice and BrdU labeling was only measured in strong labeled cells. We observed the birth of $\mathrm{NeuN}^{+}$neurons simultaneously in the deeper layers, LV/LVI as early as E10.5 with a peak occurring at E12.5 (Figure 7A and Supplementary Figures 5A,B). The birth of both LII and LIII NeuN+ neurons was observed later at E12.5, but interestingly, both layers were born in parallel and both peaking at E14 (Figure 7A and Supplementary Figures 5A,B). The birth 


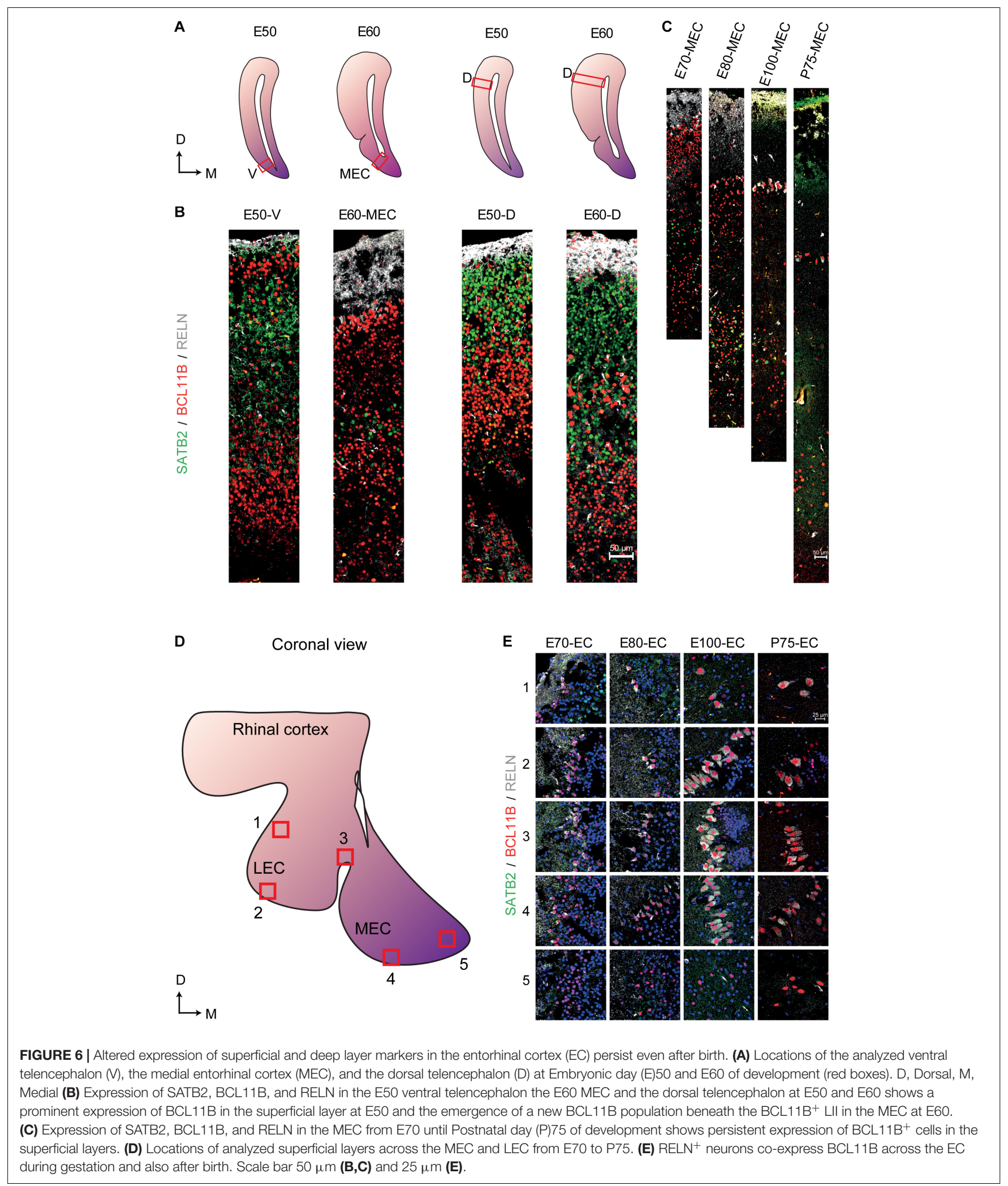

of LV/VI neurons was mostly complete by E15 and the birth of LII and LIII neurons was complete by E16 (Figure 7A and Supplementary Figures 5A,B). Interestingly, at E12.5, the BrDU labeling was weaker in the superficial neurons suggesting that they formed after the birth of the deep layer neurons and likely arise from a common progenitor (Supplementary Figure 5). 
Collectively, our findings show that LII and LIII neurons are born after the deeper layer neurons, however, the deeper layers LV/LVI arise in parallel and the superficial layers LII and LIII also arise in parallel as opposed to an inside-out deposition of layers. We therefore name this lamination process parallel lamination.

Similar to the pig, we found that postnatally in mice (P7), cells in both LII/LIII and LV co-expressed Bcl11b and Satb2 (Figures 7B-D). Interestingly, a mixture of populations was present in the $\mathrm{LV}$, including $\mathrm{Bcll1b}^{+} / \mathrm{Satb} 2^{-}$, Satb2 $2^{-} / \mathrm{Bcll1b}^{-}$ and $\mathrm{Bcll1b}^{+} / \mathrm{Satb}^{+}$cells (Figures $7 \mathbf{B}, \mathbf{D}$ ). LVI cells were $\mathrm{Bcl11b}^{+} / \mathrm{Satb}^{-}$(Figure 7B). We only observed a single population of Satb2 ${ }^{+} / \mathrm{Bcll}_{1} \mathrm{~b}^{+}$cells in LII/LIII. To investigate $\operatorname{Reln}^{+}$neurons in LII in more detail, we investigated the expression of Reln, Bcl11b and BrdU at both E12.5 and E15. We identified, most Reln ${ }^{+} / \mathrm{Bcl}_{11} \mathrm{~b}^{+}$neurons in LII were born at E12.5, however, a few were also born later at E15 (Figure 7E). A small number of Reln ${ }^{+} / \mathrm{Bcll} 1 \mathrm{~b}^{-}$neurons were born both at E12.5 and E15 (Figure 7E). A small subset of Reln ${ }^{-} /$Bcl11b $^{+}$ neurons were born both at E12.5 and at E15 (Figure 7E). This indicates that the most prominent population of neurons born at E12.5 in LII is the Reln ${ }^{+} / \mathrm{Bcll1}^{+} \mathrm{b}^{+}$neurons. Together this identifies two Reln ${ }^{+}$populations emerging at LII.

\section{DISCUSSION}

We present the boundaries of the MEC and LEC in the developing pig brain and use the pig as a model to investigate neurogenesis and lamination in the MEC. We have found it to be an excellent choice for studying brain development since it closely represents human neurogenesis. We identified that neurogenesis in the pig EC spans from > E26 to E70 (during the 1st and early 2 nd trimester) which corresponds closely to the timing of neurogenesis in humans (gestational week 7-17) (Kostovic et al., 2019) but is dissimilar to that in mice, in which it extends almost until birth (E11-E17) (Casarosa et al., 1999; Roy et al., 2004; Stagni et al., 2015). The anatomical orientation of the pig EC was also similar to that in the developing human brain as we could better view the EC in the coronal plane, similar to the human. In contrast, the EC in rodents is best viewed in the sagittal and horizontal plane. The MRI studies on the developing porcine brain showed the EC grew at a faster rate at E70 compared to the rest of the neocortex, suggesting corticogenesis timing differs in the EC than the remaining neocortex. Further, the connectivity of the postnatal EC to the peri- and post-rhinal cortex concurs with previous studies reported in the rat (Insausti et al., 1997; Burwell and Amaral, 1998). Interestingly, the total tract numbers within the LEC fluctuated between the brains more so, than the MEC and this variation might be due to individual variability. Future studies investigating how connectivity is shaped during the development of the EC would be of interest.

Immunohistochemical analyses revealed that the pig has a moderate OSVZ present from E33 to E60 in the EC, which changed in its distribution of intermediate progenitor populations, from a mixed identity of $\mathrm{EOMES}^{+} / \mathrm{PAX}^{-}$and EOMES $^{+} / \mathrm{PAX}^{+}$to an enriched population of $\mathrm{EOMES}^{+} / \mathrm{PAX}^{+}$ cells. The entorhinal cells in the pig emerge during the early second trimester and by E60, key cytoarchitectonic features delineate the MEC from the LEC. In humans, the emergence of the EC occurs toward the end of the first trimester with prominent entorhinal cells visible as early as 10.5 weeks post ovulation (Kostovic et al., 1993). The pig, therefore, has a larger OSVZ than rodents and the timing of neurogenesis concurs more closely to neurogenesis in the human. Given it is extremely difficult to obtain human fetal tissue during the second and third trimester, the large gyrencephalic pig brain should be considered a very good model.

Our study revealed novel features within the developing and postnatal EC. We identified pan-neuronal clustering and abundance of OPCs in the LEC and perirhinal cortex early in development at E60 in the superficial layers. The early emergence of these OPCs during gestation suggests that at least one wave of oligodendrogenesis may overlap with EC neurogenesis. In mice, the first OPCs emerge from the ventral medial ganglionic eminence at E11.5 and migrate throughout the telencephalon in a ventral to dorsal manner midway through gestation (Kessaris et al., 2006). Previous research has shown that in the piriform cortex, OPCs are highly plastic and can give rise to pyramidal neurons in the adult mouse (Guo et al., 2010). It is not clear what the fate of these OPCS are and whether they have migrated in from the ganglionic eminence or emerged locally by gliogenesis. It is also not clear what the purpose for these cells is in the superficial layers of the LEC and perirhinal cortex or how plastic they may be. We are also uncertain if this is a unique feature in the pig, or if they simply have not received much attention in other species. Immature neural progenitors with OPC-like anatomy have been identified in the SVZ, gray matter of the SVZ and ependymal layers within some brain regions such as the amygdala (Yachnis et al., 2000), but these differ in anatomical location to the OPCs we identified and have not been found in the superficial layers. Further investigation into the role of these clustered OPCs will help to reveal their role and function.

The EC forms part of the periarchicortex and acts as a transitional zone between the allocortex and neocortex. Understanding how it forms may provide insight into the divergence of brain region formation. We unequivocally show that the deep layer neurons are the first neurons to be born in the EC at E10.5 in the mouse. We identified that the MEC forms by parallel lamination events, that differs from classical inside-out lamination events in the neocortex. Similar to the neocortex, the deeper layers precede the birth of the superficial layers, however, both the deeper layers arise in parallel and the superficial layers also arise in parallel. The neurons are scattered evenly across LV/LVI, as well as LII/III. It is not clear what signaling mechanisms may drive this unique parallel lamination patterning in the MEC and whether this is also the case for the LEC and the other periarchicortex regions, such as the presubiculum and parasubiculum. Reln-signaling from the Cajul Retzius cells plays an important role in lamination of the neocortex (D'Arcangelo et al., 1995; Goldowitz et al., 1997) and investigation of how Cajul Retzius cells differ in their signaling in the EC or whether early born Reln ${ }^{+}$neurons contribute to the lamination patterning of the EC would be interesting to follow up in future studies. Ikaros-signaling may also be implicated since it regulates the 


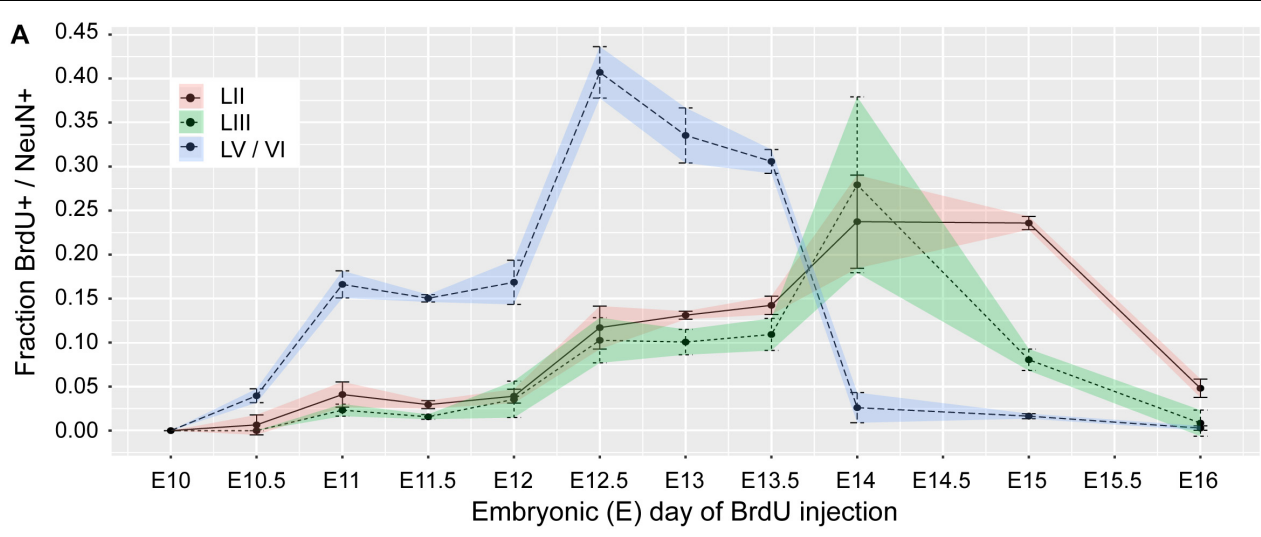

B Satb2
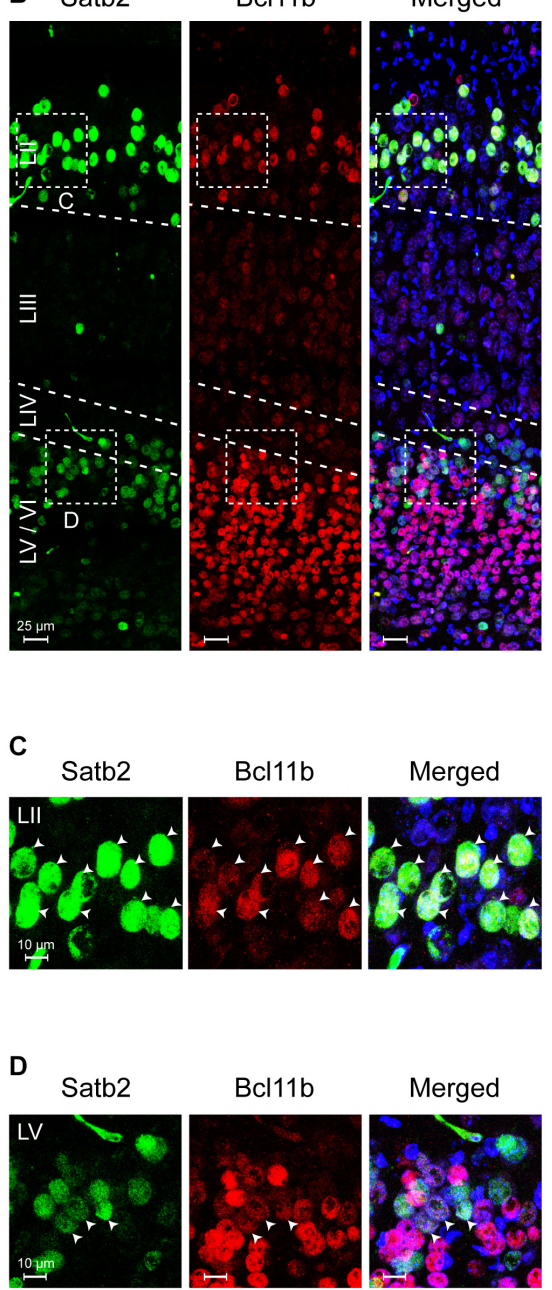

E
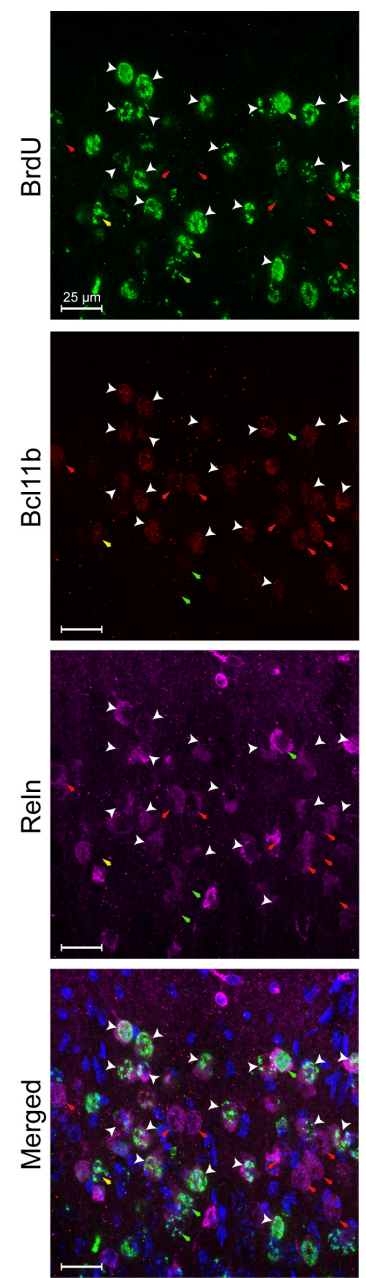

E15
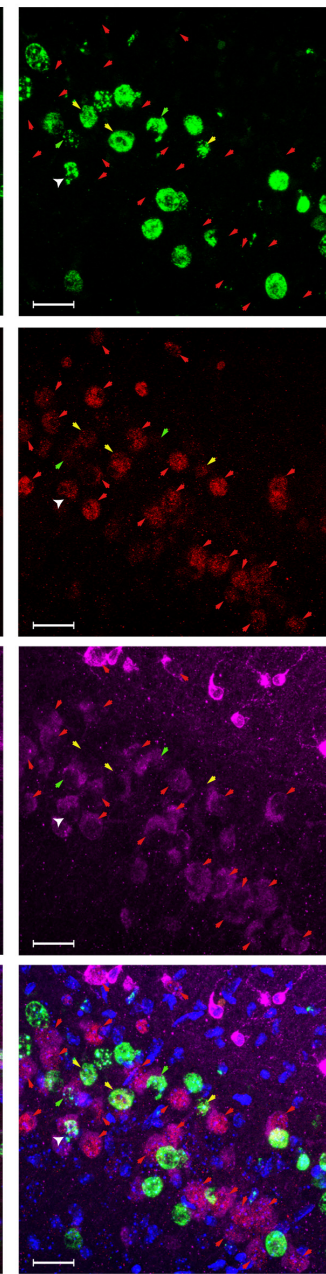

FIGURE 7 The medial entorhinal cortex (MEC) emerges via parallel lamination events. Birth timing of layers in the MEC was established using Bromodeoxyuridine (BrdU) labeling of NeuN-positive (+) neurons born at embryonic day (E)10 to E16 and identification of early born neurons by expression of Satb2, Bcl11b, and Reln. (A) Quantification of NeuN+ neurons labeled with BrdU in layer (L)II, LIII, and LVa/b/VI, respectively, at the time of BrdU injection, shown as correlation of

$\mathrm{BrdU}^{+} / \mathrm{NeuN}^{+}$fraction and injection time in gestation as embryonic day (E). Error bars denote the standard deviation. (B) Overview of Satb2 and Bcll1b expression in the medial entorhinal cortex at postnatal day (P)7. The upper-layer (UL) marker, Satb2 was expressed in the LII and LVa. The deep-layer (DL) marker Bcl11b, was expressed both in the DL and co-expressed with Satb2 in neurons in LII. Merged image additionally shows Hoechst (HO) staining. Scale bar $25 \mu \mathrm{m}$. High magnification of $\mathrm{UL}$ (C) and DL (D) inserts from A, show Satb2 ${ }^{+}$and Bcl11b expression in LIl and LV cells. White arrow heads point to cells with co-expression. Merged image additionally shows HO DNA labeling. Scale bar $10 \mu \mathrm{m}$. (E) Co-expression of Bcl11b and Reln in cells in the LII newborn neurons at E12.5 and E15 (assessed by BrdU labeling). White arrow heads show cells with immunoreactivity for BrdU, Bcl11b and Reln. Red arrows point to cells co- expressing Bcl11b and Reln. Yellow arrows depict expression of Bcl11b in BrdU labeled cells. Green arrows depict expression of Reln in BrdU labeled cells. Merged image shows immunoreactivity of BrdU, Bcl11b, Reln, and HO. Scale bar $25 \mu \mathrm{m}$. 
birth of deep and upper layer neurons in the cerebral cortex (Alsio et al., 2013). Single cell RNA sequencing and analysis studies of excitatory neuron cell fates in the neocortex confirm that deep layer neurons emerge first, prior to upper layer neurons (Loo et al., 2019; Fan et al., 2020). Even the recent emergence of flashtag labeling of embryonic progenitors that allows for greater precision in birthdate labeling of neurons (Govindan et al., 2018) confirms the classic inside-out lamination pattern of the neocortex (Telley et al., 2019). It is plausible to consider that altered lamination patterns in the MEC might relate to altered connectivity and a more thorough investigation of lamination events in the LEC and other periallocortex regions may help to elucidate whether this is a unique feature of the periallocortex.

Studying the emergence of entorhinal cells is interesting since temporal divergences exist in both the emergence and maturation of the cell types in at least the superficial layers. In the mouse, the stellate cells have been shown to emerge in the MEC at approximately E12, with pyramidal neurons emerging approximately 2 days later (Donato et al., 2017). Our study demonstrates that the early born entorhinal cells in the superficial layers express $\mathrm{Bcll}_{1} \mathrm{~b}^{+}$which is an oddity since Bcl11b is a well-known LV neuron marker in the neocortex. Co-expression of Bcl11b with Reln in the postnatal mouse brain confirms these neurons to be stellate cells. Bcll1b has been shown to be transiently expressed in all deep layer early born neurons, which is then downregulated in LVI neurons and remains expressed in LV neurons (Kwan et al., 2008). Furthermore, Bcl11b is a downstream effector of Fezf2 and its expression in neurons in the cerebral cortex mediates subcortical projection of axons (Chen et al., 2008). However, Reln ${ }^{+}$neurons in LII of the MEC project intracortically to the dentate gyrus and CA3 (Kitamura et al., 2014; Fuchs et al., 2016; Leitner et al., 2016). We presume therefore that their axonal projections are steered by other signaling mechanisms which may or may not relate to Fezf2 signaling. We also demonstrate in the pig model that the stellate cells are RELN ${ }^{-}$when they are first deposited in LII and LIII and switch on RELN later. This may have implications for the parallel lamination events observed in the mouse, as later-born LII neurons at E14 are able to bypass the LIII neurons and early expression of Reln in the LIII neurons might prevent such migration. It is not clear from our studies whether the early born stellate cells in mice express Reln at E14, but it would be interesting to investigate this. We demonstrate that the deep layer neurons and the early born superficial layer neurons arising at E12.5 both express Bcl11b which indicates they arise from a common progenitor. Interestingly, the BrdU labeling in the superficial neurons is weaker than the deep layer neurons and suggests these cells are born one or more cell divisions later. This indicates an important probabilistic decision event occurs in the common progenitor at E12.5. This observation supports the theory of stochastic cortical neurogenesis arising from radial glia progenitors that over time alters the molecular internal clocks within the cells (Llorca et al., 2019). It cannot explain, however, how neuron migration is directed, as the early newborn Reln(+ neurons are scattered across both LII and LIII at this time point. Our study clearly shows that in the mouse, LII is the last layer deposited during neurogenesis, which contrasts an earlier study which identified that LIII formed last in the rat, which was termed the "sandwich gradient" (Bayer, 1980b; Bayer et al., 1993). This contrasting finding might relate to species-specific differences and further investigation in a wider range of species is required to conclude whether this is the case. Further, Bayer (1980b) indeed report an temporal overlap in the emergence of LII and LIII cells even if LIII cells appear more prominent at specific time points. Further, in our study, by dividing $\mathrm{BrdU}^{+}$cells by the total $\mathrm{NeuN}^{+}$cells we provide an accurate reflection of the proportion of neurons born. It is important to also investigate lamination patterning in the LEC to form a more meaningful conclusion of general lamination patterns across the EC. We clearly identified subsets of Reln ${ }^{+}$cells that differed in their timing of emergence in LII. It is highly likely that the large population of Reln ${ }^{+} / \mathrm{Bcl}_{11 b^{+}}$neurons that were born early on at E12.5 are stellate cells, but the identity of the $\mathrm{Re}^{+} / \mathrm{Bcll}^{-} \mathrm{b}^{-}$ neurons is not clear. We speculate that these cells correspond to the intermediate stellate/pyramdidal cell described by Fuchs et al. (2016) or a second excitatory Reln ${ }^{+}$population expressing a unique enhancer from the stellate cells (Blankvoort et al., 2018). Further investigation is needed to elucidate the identity of the Reln ${ }^{+}$cell subtypes we identified in the MEC LII.

To conclude, we characterize neurogenesis events in the developing MEC, in a novel large mammalian model that closely resembles human neurogenesis. We describe for the first time, parallel lamination of the deeper layers followed by parallel lamination of the superficial layers in the MEC which differs from the classic inside-outside lamination of the neocortex. We also identify unique anatomical features in the porcine EC, including prominent clusters of OPCs in the LEC and white matter projections that extend from the EC bilaterally across the brain hemispheres. The EC accelerates markedly in growth at the end of neurogenesis at the end of the second trimester. We highlight particular dynamics in the emergence of Reln ${ }^{+}$ populations in the LII of the MEC and identify that Reln switches on later in these cells, days after the cells are born. We therefore provide detailed insight into the dynamics of the emerging EC which provides novel insight into altered cortical lamination events compared to the rest of the neocortex. Further studies investigating the mechanisms of parallel lamination may help to determine how this process occurs and whether this is a conserved feature across the periallocortex regions of the brain.

\section{DATA AVAILABILITY STATEMENT}

The datasets presented in this article are not readily available because we haven't generated large datasets. Requests to access the datasets should be directed to VH, vh@sund.ku.dk.

\section{ETHICS STATEMENT}

The animal study was reviewed and approved by the National Animal Ethics Committee of Denmark. Written informed consent was obtained from the owners for the participation of their animals in this study. 


\section{AUTHOR CONTRIBUTIONS}

YL performed immunohistochemistry on the pig brain, DTI/MRI, analyzed data, and contributed to the writing of the manuscript. TB performed histology of the pig brain, BrdU labeling, analyzed data, and contributed to the writing of the manuscript. YM performed the DTI/MRI and contributed to the analysis of the DTI data. JP contributed to immunohistochemistry of pig brain. MP contributed to the sectioning and Cresyl violet staining of the pig brain. NV performed BrdU labeling in mice. PT provided porcine fetal specimens. SS and JG supervised the project and reviewed the manuscript. $\mathrm{PH}$ supervised project and contributed to acquisition of funding and reviewed the manuscript. KK provided materials, personnel and resources for the BrDU labeling, and contributed to interpretation of the data. MW contributed to anatomical analysis of the EC in the pig and in acquisition of funding. $\mathrm{VH}$ contributed to the projects concept, methodology, formal analyses, project's resources, writing - reviewing and editing the manuscript, administered and managed the project, and acquired the project's funding. All authors read and approved the final manuscript.

\section{FUNDING}

The project was financed by the Independent Research Fund, Denmark under the grant (ID: DFF-7017-00071 and 802100048B), Lundbeck fund (R296-2018-2287) and the Innovation Foundation [Brainstem (4108-00008B)]. The Independent Research Fund, the Lundbeck foundation and the Innovation Foundation have supported toward the consumables and salaries of persons contributing to the project.

\section{ACKNOWLEDGMENTS}

We thank Per Torp Sangild for his donation of healthy adult sow brains for this study. We acknowledge the Core Facility for Integrated Microscopy, Faculty of Health and Medical Sciences, University of Copenhagen for access use of the Axio Scan Z1.

\section{SUPPLEMENTARY MATERIAL}

The Supplementary Material for this article can be found online at: https://www.frontiersin.org/articles/10.3389/fnana.2021. 663667/full\#supplementary-material

Supplementary Figure 1 | Borders of the developing porcine entorhinal cortex (EC). Cresyl violet stained 4 or 5 coronal sections of the piriform lobe from Embryonic day (E)60 to postnatal day (P)75 depicted in a rostral to caudal series. The first section is rostral to the EC, the second section depicts the LEC occupying the EC entity, the third section includes both MEC and LEC present, the fourth section depicts the MEC occupying the entire mediolateral entity and the fifth section is the most caudal part of the piriform lobe. Dentate gyrus (DG); hippocampal area (HA); amygdala (Amyg); posterior rhinal sulcus (RHP); medial entorhinal cortex (MEC); lateral entorhinal cortex (LEC); pre-subiculum (PreS); para-subiculum (PaS), subiculum (Sub); perirhinal cortex (PER). Scale bar $1 \mathrm{~cm}$.

Supplementary Figure 2 | The morphology of the entorhinal cortex at Embryonic day (E)50 to E70. Cresyl violet staining of the developing cortex show a prominent layer or entorhinal neurons with large nuclei in the superficial layer from E50 onwards whereas, the glia cells are difficult to identify at E50 from the nissl staining alone. Scale bar $200 \mu \mathrm{m}$.

Supplementary Figure $\mathbf{3} \mid$ Characterization of the germinal layers in the developing porcine entorhinal cortex (EC). (A) A schematic overview of the location of the characterized medial EC (MEC). Axes: D, dorsal; V, Ventral; A, Anterior; P, posterior, $\mathrm{M}$, medial. Orange line depicts the anatomical location of the cut sections. (B) Expression of GFAP and FABP7 in the MEC ventricular zone (VZ). Scale bar $25 \mu \mathrm{m}$. Images are enlarged from boxes shown in C. White arrows mark co-localized expression of GFAP and FABP7. (C) Temporal expression of radial glia (GFAP, FABP7, PAX6, SOX2) during MEC development. Scale bar $50 \mu \mathrm{m}$ (D). Quantification of the thickness in $\mu \mathrm{m}$ of the VZ during development. Error bars represent SD. (E) Expression of EOMES and PAX6 in the EC. White arrow heads depict EOMES ${ }^{+} / \mathrm{PAX6}^{-}$cells and asterixis depict EOMES ${ }^{+} / \mathrm{PAX6}^{+}$cells. Scale bar $25 \mu \mathrm{m}$ (up) and $50 \mu \mathrm{m}$ (bottom). (F) Quantification of the EOMES ${ }^{+} / \mathrm{PAX}^{+}$ and $\mathrm{EOMES}^{+} / \mathrm{PAX}^{-}$cell populations in the germinal zone.

(G) TBR1/EOMES/RELN expression during development. Scale bar $50 \mu \mathrm{m}$. (H) Expression of TBR1/EOMES/RELN in the marginal zone and the cortical plate, enlarged from boxes in G. Yellow circled cells are TBR1+/EOMES ${ }^{-} /$RELN $^{-}$Scale bar $25 \mu \mathrm{m}$. (HO = Hoeschst, $V=$ ventral telencephalon).

Supplementary Figure 4 | Comparative expression of superficial and deep layer markers in the early developing entorhinal cortex (EC) vs. the dorsal, cingulate gyrus. Expression of the canonical deep layer marker (BCL11B), superficial layer marker (SATB2) and stellate cell/Cajal-Retzius cells (CR cells) marker RELN from Embryonic day (E)26 to E39 shows the prevalence of BCL11B and SATB2 from E33 onwards in the superficial marginalzone/cortical plate and the absence of RELN at these time points within the developing cortical plate. Scale bar $50 \mu \mathrm{m}$.

Supplementary Figure $\mathbf{5} \mid$ Laminar birth dating of the medial entorhinal cortex (MEC) in the mouse. Bromodeoxyuri-dine (BrdU) labeling in combination with analysis of NeuN expression of postnatal day (P)7 MEC shows the emergence of newborn neurons from embryonic day (E)10 to E16. (A) Representative z-stack images from BrdU injections from E9-E16. Dotted lines represent the LII/LIII boundary and the LIV lamina dissecans. Insert boxes are shown at higher magnification in B. White arrowheads depict cells highlighted in B. Scale bar 25 $\mu \mathrm{m}$. (B) High magnification of representative BrdU labeled cells with overlapping expression of NeuN in a single z-plane from LII and LIII areas in A. White arrows highlight BrdU labeled cells. Yellow asterix $\left(^{*}\right)$ denotes cells with strong BrdU labeling which were considered to be born at the time of BrdU injection. Scale bar $10 \mu \mathrm{m}$.

Supplementary Table 1 | Overview of porcine brains and brain sections used in the study.

Supplementary Table 2 | Overview of mouse brains, litters and sections used in the study.

Supplementary Table 3 | Overview of primary antibodies and dilutions used.

Supplementary Table 4 | Overview of secondary and negative control antibodies and dilutions used.

Supplementary Table $\mathbf{5}$ | Raw data for quantification of neurons in mouse brains.

Supplementary Table 6 | Connectivity of neuronal tracts to/from the lateral entorhinal cortex (LEC) and the medial entorhinal cortex (MEC) in the pig at postnatal day $(P) 75$. The average connectivity from three analyzed brains is shown with the standard deviation in brackets. Abbreviations: AM, amygdala; $\mathrm{CN}$, caudate nucleus, DG, dentate gyrus; HC, dorsal hippocampal commissure; LEC, lateral entorhinal cortex; LOT, lateral olfactory tract; MEC, medial entorhinal cortex; Nucleus accumbens, NAc; PUT, putamen; SUB, subiculum. 


\section{REFERENCES}

Alsio, J. M., Tarchini, B., Cayouette, M., and Livesey, F. J. (2013). Ikaros promotes early-born neuronal fates in the cerebral cortex. Proc. Natl. Acad. Sci. U.S.A. 110, E716-E725.

Arnold, S. J., Huang, G. J., Cheung, A. F., Era, T., Nishikawa, S., Bikoff, E. K., et al. (2008). The T-box transcription factor Eomes/Tbr2 regulates neurogenesis in the cortical subventricular zone. Genes Dev. 22, 2479-2484. doi: 10.1101/gad. 475408

Bayer, S. A. (1980a). Development of the hippocampal region in the rat. I. Neurogenesis examined with $3 \mathrm{H}$-thymidine autoradiography. J. Comp. Neurol. 190, 87-114. doi: 10.1002/cne.901900107

Bayer, S. A. (1980b). Development of the hippocampal region in the rat. II. Morphogenesis during embryonic and early postnatal life. J. Comp. Neurol. 190, 115-134. doi: 10.1002/cne.901900108

Bayer, S. A., Altman, J., Russo, R. J., and Zhang, X. (1993). Timetables of neurogenesis in the human brain based on experimentally determined patterns in the rat. Neurotoxicology 14, 83-144.

Bergmann, E., Zur, G., Bershadsky, G., and Kahn, I. (2016). The organization of mouse and human cortico-hippocampal networks estimated by intrinsic functional connectivity. Cereb. Cortex 26, 4497-4512. doi: 10.1093/cercor/ bhw327

Bjarkam, C. R., Glud, A. N., Orlowski, D., Sorensen, J. C. H., and Palomero-Gallagher, N. (2017). The telencephalon of the Gottingen minipig, cytoarchitecture and cortical surface anatomy. Brain Struct. Funct. 222, 20932114. doi: 10.1007/s00429-016-1327-5

Blankvoort, S., Witter, M. P., Noonan, J., Cotney, J., and Kentros, C. (2018). Marked diversity of unique cortical enhancers enables neuron-specific tools by enhancer-driven gene expression. Curr. Biol. 28(13) 210:e2105.

Boccara, C. N., Kjonigsen, L. J. I, Hammer, M., Bjaalie, J. G., Leergaard, T. B., and Witter, M. P. (2015). A three-plane architectonic atlas of the rat hippocampal region. Hippocampus 25, 838-857. doi: 10.1002/hipo.22407

Burwell, R. D., and Amaral, D. G. (1998). Perirhinal and postrhinal cortices of the rat: interconnectivity and connections with the entorhinal cortex. J. Comp. Neurol. 391, 293-321. doi: 10.1002/(sici)1096-9861(19980216)391:3<293::aidcne2>3.0.co; $2-\mathrm{x}$

Canto, C. B., and Witter, M. P. (2012a). Cellular properties of principal neurons in the rat entorhinal cortex. I. The lateral entorhinal cortex. Hippocampus 22, 1256-1276. doi: 10.1002/hipo.20997

Canto, C. B., and Witter, M. P. (2012b). Cellular properties of principal neurons in the rat entorhinal cortex. II. The medial entorhinal cortex. Hippocampus 22, 1277-1299. doi: 10.1002/hipo.20993

Casarosa, S., Fode, C., and Guillemot, F. (1999). Mash1 regulates neurogenesis in the ventral telencephalon. Development 126, 525-534.

Chen, B., Wang, S. S., Hattox, A. M., Rayburn, H., Nelson, S. B., and McConnell, S. K. (2008). The Fezf2-Ctip2 genetic pathway regulates the fate choice of subcortical projection neurons in the developing cerebral cortex. Proc. Natl. Acad. Sci. U.S.A. 105, 11382-11387. doi: 10.1073/pnas.0804918105

Conrad, M. S., Dilger, R. N., and Johnson, R. W. (2012). Brain growth of the domestic pig (Sus scrofa) from 2 to 24 weeks of age: a longitudinal MRI study. Dev. Neurosci. 34, 291-298. doi: 10.1159/000339311

Conrad, M. S., Sutton, B. P., Dilger, R. N., and Johnson, R. W. (2014). An in vivo three-dimensional magnetic resonance imaging-based averaged brain collection of the neonatal piglet (Sus scrofa). PLoS One 9:e107650. doi: 10.1371/ journal.pone.0107650

Cooper, J. A. (2008). A mechanism for inside-out lamination in the neocortex. Trends Neurosci. 31, 113-119. doi: 10.1016/j.tins.2007.12.003

D’Arcangelo, G., Miao, G. G., Chen, S. C., Soares, H. D., Morgan, J. I., and Curran, T. (1995). A protein related to extracellular matrix proteins deleted in the mouse mutant reeler. Nature 374, 719-723. doi: 10.1038/374719a0

Dolorfo, C. L., and Amaral, D. G. (1998). Entorhinal cortex of the rat: organization of intrinsic connections. J. Comp. Neurol. 398, 49-82. doi: 10.1002/(sici)10969861(19980817)398:1<49::aid-cne4>3.0.co;2-9

Domnisoru, C., Kinkhabwala, A. A., and Tank, D. W. (2013). Membrane potential dynamics of grid cells. Nature 495, 199-204. doi: 10.1038/nature11973

Donato, F., Jacobsen, R. I., Moser, M. B., and Moser, E. I. (2017). Stellate cells drive maturation of the entorhinal-hippocampal circuit. Science 355, eaai8178. doi: $10.1126 /$ science.aai8178
Fan, X., Fu, Y., Zhou, X., Sun, L., Yang, M., Wang, M., et al. (2020). Singlecell transcriptome analysis reveals cell lineage specification in temporal-spatial patterns in human cortical development. Sci. Adv. 6:eaaz2978. doi: 10.1126/ sciadv.aaz2978

Farr, M., Kitas, G. D., Waterhouse, L., Jubb, R., Felix-Davies, D., and Bacon, P. A. (1988). Treatment of psoriatic arthritis with sulphasalazine: a one year open study. Clin. Rheumatol. 7, 372-377. doi: 10.1007/bf02239195

Fietz, S. A., Kelava, I., Vogt, J., Wilsch-Brauninger, M., Stenzel, D., Fish, J. L., et al. (2010). OSVZ progenitors of human and ferret neocortex are epithelial-like and expand by integrin signaling. Nat. Neurosci. 13, 690-699. doi: 10.1038/nn.2553

Fuchs, E. C., Neitz, A., Pinna, R., Melzer, S., Caputi, A., and Monyer, H. (2016). Local and distant input controlling excitation in layer II of the medial entorhinal cortex. Neuron 89, 194-208. doi: 10.1016/j.neuron.2015.11.029

Garcia, A. D., and Buffalo, E. A. (2020). Anatomy and function of the primate entorhinal cortex. Annu. Rev. Vis. Sci. 6, 411-432. doi: 10.1146/annurev-vision030320-041115

Garcia-Cabezas, M. A., John, Y. J., Barbas, H., and Zikopoulos, B. (2016). Distinction of neurons, glia and endothelial cells in the cerebral cortex: an algorithm based on cytological features. Front. Neuroanat. 10:107. doi: 10.3389/ fnana.2016.00107

Gatome, C. W., Slomianka, L., Lipp, H. P., and Amrein, I. (2010). Number estimates of neuronal phenotypes in layer II of the medial entorhinal cortex of rat and mouse. Neuroscience 170, 156-165. doi: 10.1016/j.neuroscience.2010. 06.048

Goldowitz, D., Cushing, R. C., Laywell, E., D’Arcangelo, G., Sheldon, M., Sweet, H. O., et al. (1997). Cerebellar disorganization characteristic of reeler in scrambler mutant mice despite presence of reelin. J. Neurosci. 17, 8767-8777. doi: 10.1523/jneurosci.17-22-08767.1997

Govindan, S., Oberst, P., and Jabaudon, D. (2018). In vivo pulse labeling of isochronic cohorts of cells in the central nervous system using FlashTag. Nat. Protoc. 13, 2297-2311. doi: 10.1038/s41596-018-0038-1

Guo, F., Maeda, Y., Ma, J., Xu, J., Horiuchi, M., Miers, L., et al. (2010). Pyramidal neurons are generated from oligodendroglial progenitor cells in adult piriform cortex. J. Neurosci. 30, 12036-12049. doi: 10.1523/jneurosci.1360-10.2010

Hevner, R. F., Neogi, T., Englund, C., Daza, R. A., and Fink, A. (2003). Cajal-Retzius cells in the mouse: transcription factors, neurotransmitters, and birthdays suggest a pallial origin. Brain Res. Dev. Brain Res. 141, 39-53. doi: 10.1016/ s0165-3806(02)00641-7

Holm, I. E., and Geneser, F. A. (1989). Histochemical demonstration of zinc in the hippocampal region of the domestic pig: I. Entorhinal area, parasubiculum, and presubiculum. J. Comp. Neurol. 287, 145-163. doi: 10.1002/cne.902870202

Insausti, R., Herrero, M. T., and Witter, M. P. (1997). Entorhinal cortex of the rat: cytoarchitectonic subdivisions and the origin and distribution of cortical efferents. Hippocampus 7, 146-183. doi: 10.1002/(sici)1098-1063(1997) 7:2<146::aid-hipo4>3.0.co;2-1

Insausti, R., Munoz-Lopez, M., Insausti, A. M., and Artacho-Perula, E. (2017). The human periallocortex: layer pattern in presubiculum, parasubiculum and entorhinal cortex. a review. Front. Neuroanat. 11:84. doi: 10.3389/fnana.2017. 00084

Insausti, R., Tunon, T., Sobreviela, T., Insausti, A. M., and Gonzalo, L. M. (1995). The human entorhinal cortex: a cytoarchitectonic analysis. J. Comp. Neurol. 355, 171-198. doi: 10.1002/cne.903550203

Jelsing, J., Nielsen, R., Olsen, A. K., Grand, N., Hemmingsen, R., and Pakkenberg, B. (2006). The postnatal development of neocortical neurons and glial cells in the Gottingen minipig and the domestic pig brain. J. Exp. Biol. 209(Pt 8), 1454-1462. doi: 10.1242/jeb.02141

Jukic, A. M., Baird, D. D., Weinberg, C. R., McConnaughey, D. R., and Wilcox, A. J. (2013). Length of human pregnancy and contributors to its natural variation. Hum. Reprod. 28, 2848-2855. doi: 10.1093/humrep/det297

Kessaris, N., Fogarty, M., Iannarelli, P., Grist, M., Wegner, M., and Richardson, W. D. (2006). Competing waves of oligodendrocytes in the forebrain and postnatal elimination of an embryonic lineage. Nat. Neurosci. 9, 173-179. doi: $10.1038 / \mathrm{nn} 1620$

Kim, S. W. (2010). Recent advances in sow nutrition. Rev. Brasil.Zoot. 39, 303-310. doi: 10.1590/s1516-35982010001300033

Kitamura, T., Pignatelli, M., Suh, J., Kohara, K., Yoshiki, A., Abe, K., et al. (2014). Island cells control temporal association memory. Science 343, 896-901. doi: $10.1126 /$ science. 1244634 
Kolenkiewicz, M., Robak, A., Rowniak, M., Bogus-Nowakowska, K., Calka, J., and Majewski, M. (2009). Distribution of cocaine- and amphetamine-regulated transcript in the hippocampal formation of the guinea pig and domestic pig. Folia Morphol. (Warsz) 68, 23-31.

Kostovic, I., Petanjek, Z., and Judas, M. (1993). Early areal differentiation of the human cerebral cortex: entorhinal area. Hippocampus 3, 447-458. doi: 10.1002/ hipo.450030406

Kostovic, I., Sedmak, G., and Judas, M. (2019). Neural histology and neurogenesis of the human fetal and infant brain. Neuroimage 188, 743-773. doi: 10.1016/j. neuroimage.2018.12.043

Kumar, S., and Hedges, S. B. (1998). A molecular timescale for vertebrate evolution. Nature 392, 917-920. doi: 10.1038/31927

Kwan, K. Y., Lam, M. M., Krsnik, Z., Kawasawa, Y. I., Lefebvre, V., and Sestan, N. (2008). SOX5 postmitotically regulates migration, postmigratory differentiation, and projections of subplate and deep-layer neocortical neurons. Proc. Natl. Acad. Sci. U.S.A. 105, 16021-16026. doi: 10.1073/pnas.0806791105

Leitner, F. C., Melzer, S., Lutcke, H., Pinna, R., Seeburg, P. H., Helmchen, F., et al. (2016). Spatially segregated feedforward and feedback neurons support differential odor processing in the lateral entorhinal cortex. Nat. Neurosci. 19, 935-944. doi: 10.1038/nn.4303

Llorca, A., Ciceri, G., Beattie, R., Wong, F. K., Diana, G., Serafeimidou-Pouliou, E., et al. (2019). A stochastic framework of neurogenesis underlies the assembly of neocortical cytoarchitecture. Elife 8:e51381.

Loo, L., Simon, J. M., Xing, L., McCoy, E. S., Niehaus, J. K., Guo, J., et al. (2019). Single-cell transcriptomic analysis of mouse neocortical development. Nat. Commun. 10:134.

Martinez-Cerdeno, V., Cunningham, C. L., Camacho, J., Antczak, J. L., Prakash, A. N., Cziep, M. E., et al. (2012). Comparative analysis of the subventricular zone in rat, ferret and macaque: evidence for an outer subventricular zone in rodents. PLoS One 7:e30178. doi: 10.1371/journal.pone.0030178

Mufson, E. J., Brady, D. R., and Kordower, J. H. (1990). Tracing neuronal connections in postmortem human hippocampal complex with the carbocyanine dye DiI. Neurobiol. Aging 11, 649-653. doi: 10.1016/01974580(90)90031-t

Patten, A. R., Fontaine, C. J., and Christie, B. R. (2014). A comparison of the different animal models of fetal alcohol spectrum disorders and their use in studying complex behaviors. Front. Pediatr. 2:93. doi: 10.3389/fped.2014.00093

Pontelo, T. P., Miranda, J. R., Felix, M. A. R., Pereira, B. A., da Silva, W. E., Avelar, G. F., et al. (2018). Histological characteristics of the gonads of pig fetuses and their relationship with fetal anatomical measurements. Res. Vet. Sci. 117, 28-36. doi: 10.1016/j.rvsc.2017.11.005

Ray, S., and Brecht, M. (2016). Structural development and dorsoventral maturation of the medial entorhinal cortex. Elife 5:e13343.

Rowland, D. C., Obenhaus, H. A., Skytoen, E. R., Zhang, Q., Kentros, C. G., Moser, E. I., et al. (2018). Functional properties of stellate cells in medial entorhinal cortex layer II. Elife 7:e36664.

Rowland, D. C., Weible, A. P. I, Wickersham, R., Wu, H., Mayford, M., Witter, M. P., et al. (2013). Transgenically targeted rabies virus demonstrates a major monosynaptic projection from hippocampal area CA2 to medial entorhinal layer II neurons. J. Neurosci. 33, 14889-14898. doi: 10.1523/jneurosci.104613.2013

Roy, K., Kuznicki, K., Wu, Q., Sun, Z., Bock, D., Schutz, G., et al. (2004). The Tlx gene regulates the timing of neurogenesis in the cortex. J. Neurosci. 24, 8333-8345. doi: 10.1523/jneurosci.1148-04.2004

Saikali, S., Meurice, P., Sauleau, P., Eliat, P. A., Bellaud, P., Randuineau, G., et al. (2010). A three-dimensional digital segmented and deformable brain atlas of the domestic pig. J. Neurosci. Methods 192, 102-109. doi: 10.1016/j.jneumeth. 2010.07.041

Sansom, S. N., Griffiths, D. S., Faedo, A., Kleinjan, D. J., Ruan, Y., Smith, J., et al. (2009). The level of the transcription factor Pax6 is essential for controlling the balance between neural stem cell self-renewal and neurogenesis. PLoS Genet 5:e1000511. doi: 10.1371/journal.pgen.1000511

Schmidt-Hieber, C., and Hausser, M. (2013). Cellular mechanisms of spatial navigation in the medial entorhinal cortex. Nat. Neurosci. 16, 325-331. doi: 10.1038/nn.3340

Schultz, H., Sommer, T., and Peters, J. (2015). The role of the human entorhinal cortex in a representational account of memory. Front. Hum. Neurosci. 9:628. doi: 10.3389/fnhum.2015.00628
Smart, I. H., Dehay, C., Giroud, P., Berland, M., and Kennedy, H. (2002). Unique morphological features of the proliferative zones and postmitotic compartments of the neural epithelium giving rise to striate and extrastriate cortex in the monkey. Cereb. Cortex 12, 37-53. doi: 10.1093/cercor/ 12.1.37

Stagni, F., Giacomini, A., Guidi, S., Ciani, E., and Bartesaghi, R. (2015). Timing of therapies for Down syndrome: the sooner, the better. Front. Behav. Neurosci. 9:265. doi: 10.3389/fnbeh.2015.00265

Staubli, U., Ivy, G., and Lynch, G. (1984). Hippocampal denervation causes rapid forgetting of olfactory information in rats. Proc. Natl. Acad. Sci. U.S.A. 81, 5885-5887. doi: 10.1073/pnas.81.18.5885

Stephan, H. (1975). "Allocortex," in Handbuch der Mikroskopischen Anatomie des Menschen, ed. W. H. Bargmann (New York, NY: Springer), S1-S998.

Stephan, H. (1983). Evolutionary trend in limbic structures. Neurosci. Behav. Rev. 7, 367-374. doi: 10.1016/0149-7634(83)90041-6

Stephan, H., and Andy, O. J. (1970). “The allocortex in primates," in Advances in Primatology, eds C. R. Noback and W. Montagna (New York, NY: Appleton Century Croft), 289-297.

Sun, Y., Nguyen, A. Q., Nguyen, J. P., Le, L., Saur, D., Choi, J., et al. (2014). Cell-type-specific circuit connectivity of hippocampal CA1 revealed through Cre-dependent rabies tracing. Cell Rep. 7, 269-280. doi: 10.1016/j.celrep.2014. 02.030

Telley, L., Agirman, G., Prados, J., Amberg, N., Fievre, S., Oberst, P., et al. (2019). Temporal patterning of apical progenitors and their daughter neurons in the developing neocortex. Science 364:eaav2522. doi: 10.1126/science. aav2522

Totterdell, S., and Meredith, G. E. (1997). Topographical organization of projections from the entorhinal cortex to the striatum of the rat. Neuroscience 78, 715-729. doi: 10.1016/s0306-4522(96)00592-1

Vandrey, B., Garden, D. L. F., Ambrozova, V., McClure, C., Nolan, M. F., and Ainge, J. A. (2020). Fan cells in layer 2 of the lateral entorhinal cortex are critical for episodic-like memory. Curr. Biol. 30(1) 16:e165.

Vasistha, N. A., Johnstone, M., Barton, S. K., Mayerl, S. E., Thangaraj Selvaraj, B., Thomson, P. A., et al. (2019). Familial $t(1 ; 11)$ translocation is associated with disruption of white matter structural integrity and oligodendrocyte-myelin dysfunction. Mol. Psychiatry 24, 1641-1654.

Wernersson, R., Schierup, M. H., Jorgensen, F. G., Gorodkin, J., Panitz, F., Staerfeldt, H. H., et al. (2005). Pigs in sequence space: a $0.66 \mathrm{X}$ coverage pig genome survey based on shotgun sequencing. BMC Genomics 6:70. doi: 10 . 1186/1471-2164-6-70

Wilhite, B. L., Teyler, T. J., and Hendricks, C. (1986). Functional relations of the rodent claustral-entorhinal-hippocampal system. Brain Res. 365, 54-60. doi: 10.1016/0006-8993(86)90721-3

Winter, J. D., Dorner, S., Lukovic, J., Fisher, J. A., St Lawrence, K. S., and Kassner, A. (2011). Noninvasive MRI measures of microstructural and cerebrovascular changes during normal swine brain development. Pediatr. Res. 69(5 Pt 1), 418-424. doi: 10.1203/pdr.0b013e31821 $10 \mathrm{f} 7 \mathrm{e}$

Witter, M. P., Doan, T. P., Jacobsen, B., Nilssen, E. S., and Ohara, S. (2017). Architecture of the entorhinal cortex a review of entorhinal anatomy in rodents with some comparative notes. Front. Syst. Neurosci. 11:46. doi: 10.3389/fnsys. 2017.00046

Witter, M. P., Room, P., Groenewegen, H. J., and Lohman, A. H. (1986). Connections of the parahippocampal cortex in the cat. V. Intrinsic connections; comments on input/output connections with the hippocampus. J. Comp. Neurol. 252, 78-94. doi: 10.1002/cne.902520105

Wouterlood, F. G., Hartig, W., Bruckner, G., and Witter, M. P. (1995). Parvalbumin-immunoreactive neurons in the entorhinal cortex of the rat: localization, morphology, connectivity and ultrastructure. J. Neurocytol. 24, 135-153. doi: $10.1007 /$ bf01181556

Woznicka, A., Malinowska, M., and Kosmal, A. (2006). Cytoarchitectonic organization of the entorhinal cortex of the canine brain. Brain Res. Rev. 52, 346-367. doi: 10.1016/j.brainresrev.2006.04.008

Wyss, J. M., Sripanidkulchai, B., and Hickey, T. L. (1983). An analysis of the time of origin of neurons in the entorhinal and subicular cortices of the cat. J. Comp. Neurol. 221, 341-357. doi: 10.1002/cne.902210309

Yachnis, A. T., Roper, S. N., Love, A., Fancey, J. T., and Muir, D. (2000). Bcl-2 immunoreactive cells with immature neuronal phenotype exist in the 
nonepileptic adult human brain. J. Neuropathol. Exp. Neurol. 59, 113-119. doi: 10.1093/jnen/59.2.113

Yushkevich, P. A., Piven, J., Hazlett, H. C., Smith, R. G., Ho, S., Gee, J. C., et al. (2006). User-guided 3D active contour segmentation of anatomical structures: significantly improved efficiency and reliability. Neuroimage 31, 1116-1128. doi: 10.1016/j.neuroimage.2006.01.015

Zykin, P. A. I, Moiseenko, A., Tkachenko, L. A., Nasyrov, R. A., Tsvetkov, E. A., and Krasnoshchekova, E. I. (2018). Peculiarities of Cyto- and chemoarchitectonics of human entorhinal cortex during the fetal period. Bull. Exp. Biol. Med. 164, 497-501. doi: 10.1007/s10517-0184020-2
Conflict of Interest: The authors declare that the research was conducted in the absence of any commercial or financial relationships that could be construed as a potential conflict of interest.

Copyright (C) 2021 Liu, Bergmann, Mori, Peralvo Vidal, Pihl, Vasistha, Thomsen, Seemann, Gorodkin, Hyttel, Khodosevich, Witter and Hall. This is an open-access article distributed under the terms of the Creative Commons Attribution License (CC BY). The use, distribution or reproduction in other forums is permitted, provided the original author(s) and the copyright owner(s) are credited and that the original publication in this journal is cited, in accordance with accepted academic practice. No use, distribution or reproduction is permitted which does not comply with these terms. 